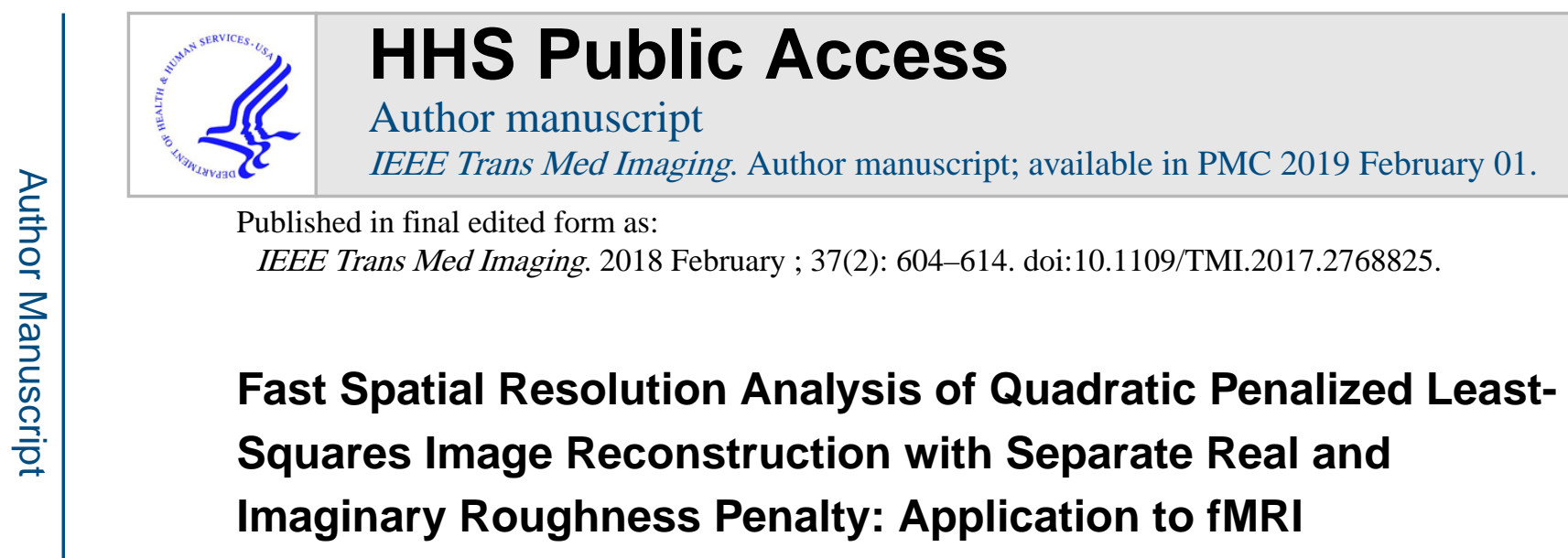

\author{
Valur T. Olafsson [Member, IEEE], \\ Northeastern University, Boston, MA 02115 USA
}

Douglas C. Noll [Senior Member, IEEE], and

Biomedical Engineering Department, The University of Michigan, Ann Arbor, MI 48109-2099 USA

Jeffrey A. Fessler [Fellow, IEEE]

Department of Electrical Engineering and Computer Science, The University of Michigan, Ann Arbor, MI 48109-2122 USA

\begin{abstract}
Penalized least-squares iterative image reconstruction algorithms used for spatial resolution limited imaging, such as functional magnetic resonance imaging (fMRI), commonly use a quadratic roughness penalty to regularize the reconstructed images. When used for complexvalued images, the conventional roughness penalty regularizes the real and imaginary parts equally. However, these imaging methods sometimes benefit from separate penalties for each part. The spatial smoothness from the roughness penalty on the reconstructed image is dictated by the regularization parameter(s). One method to set the parameter to a desired smoothness level is to evaluate the full width at half maximum (FWHM) of the reconstruction method's local impulse response. Previous work has shown that when using the conventional quadratic roughness penalty one can approximate the local impulse response using an FFT-based calculation. However, that acceleration method cannot be applied directly for separate real and imaginary regularization. This paper proposes a fast and stable calculation for this case that also uses FFT-based calculations to approximate the local impulse responses of the real and imaginary parts. This approach is demonstrated with a quadratic image reconstruction of fMRI data that uses separate roughness penalties for the real and imaginary parts.
\end{abstract}

\title{
Index Terms
}

Local impulse response; local point spread function (LPSF); quadratic penalized least-squares (QPLS); separate real and imaginary regularization; magnetic resonance imaging (MRI); functional MRI (fMRI)

Personal use of this material is permitted. However, permission to use this material for any other purposes must be obtained from the IEEE by sending a request to pubs-permissions@ieee.org. 


\section{Introduction}

Iterative image reconstruction methods are growing in popularity for many imaging problems. These methods usually involve iteratively optimizing a cost function that fits the acquired data to a linear physics-based signal model. Since measured data is contaminated with noise, the reconstructed images can have errors due to noise if the problem is badly conditioned. One way to improve the conditioning and thus reduce variance is to add a regularization term to the cost function [1], at a price of additional bias to the reconstructed image.

Nonquadratic, nonsmooth, or sparsifying regularizers, such as $\ell_{1}$ regularizers, have become increasingly popular. These types of regularizers are designed to maintain spatially sharp image transitions and contrast, such as edges. Hence, they are more effective for highresolution imaging applications, such as structural and anatomical imaging in MRI [2]. Functional magnetic resonance imaging (fMRI), with its mechanistic partial volume effect and weak functional contrast, may not benefit from such regularizers [3]. This work focuses on quadratic roughness penalties that are commonly used for fMRI, and where the bias is a reduction in spatial resolution.

For complex-valued images, conventional quadratic roughness penalties affect the real and imaginary parts equally. Sometimes, unconventional roughness penalties are needed, for example in separate regularization of magnitude and phase [4]-[6] or real and imaginary parts, which is the topic of this paper. This type of regularization has been used in digital holography [7], functional magnetic resonance imaging (fMRI) [8] and partial Fourier imaging [9].

The regularization parameter determines how smooth the reconstructed image becomes and it can be challenging to choose this parameter appropriately. One method is to base that choice on a user-defined desired spatial resolution for the reconstructed image [10], as opposed to standard MSE-based model selection methods such as cross-validation or SURE [11]. Spatial resolution can be quantified using the full width at half maximum (FWHM) of the reconstructions impulse response or, as it is also called, point spread function (PSF). To find the value of a regularization parameter that provides the desired resolution, one can tabulate the FWHM of PSFs that result from a range of regularization parameter values [10]. Through interpolation, this table is then used to find a regularization parameter value that yields images with the desired resolution.

For computational efficiency it is more convenient if the PSF for one spatial location is indicative of all other locations. This occurs when the PSF is shift invariant, i.e., when the PSF, and hence its FWHM, is uniform across the reconstructed image. However, for many penalized image reconstruction algorithms the PSF is shift variant, which can be undesirable. By designing an appropriately spatially variant penalty [8], [10], this resolution nonuniformity can often be counteracted. However, one may still need to evaluate the PSF multiple times to find the spatially dependent regularization parameters that achieve the desired resolution. 
There have been methods proposed to design spatially varying penalties based on local impulse responses [10], [12] to correct for the spatially varying resolutions of quadratic penalized least-squares (QPLS) cost functions using conventional quadratic roughness penalties [10]. There it was shown, one can use the fast Fourier transform (FFT) to compute quickly an accurate approximation to the local impulse response [13]. However, that approach is not directly applicable to the case of separately regularizing the real and imaginary parts of the image.

This paper presents an FFT-based method to calculate an approximate local impulse response when using QPLS cost functions with separate real and imaginary quadratic roughness penalties. Section II presents the problem and the notation that is used in the paper. Section III reviews the special case where the regularizer penalizes the real and imaginary parts equally. The local impulse response for that case has previously been derived and we review the details of an FFT-based accelerated method to calculate it. This formulation motivates a similar accelerated method for the separate real and imaginary penalty, which is described in Section IV. Finally, Section V presents two examples with performance analysis of the accelerated methods and those results are discussed in Section VI.

\section{Problem Statement and Notation}

We assume the following discrete and linear noisy signal model for the acquired data vector $y$ :

$$
\boldsymbol{y}=\boldsymbol{A} \boldsymbol{x}+\varepsilon,
$$

where vector $\boldsymbol{x}$ denotes the object being imaged, system matrix $\boldsymbol{A}$ is a linear transform from image space to acquired data space, and vector $\boldsymbol{\varepsilon}$ is the additive noise. All vectors are column vectors and vectors in image space have lexicographic ordering if the imaging problem is two dimensional (2D) or higher. The number of acquired data points is $M$ and the number of voxels that are to be reconstructed is $N$. Thus, $x$ is of size $N \times 1, A$ is $M \times N$, and $\boldsymbol{y}$ and $\boldsymbol{\varepsilon}$ are $M \times 1$.

Many model-based image reconstruction methods use the signal model in (1) to facilitate the estimation of $\boldsymbol{x}$ from $\boldsymbol{y}$, as is common in MRI [14]. One such estimation method is the quadratic penalized least-squares (QPLS) estimator. It uses the model in (1) along with a quadratic penalty function $\mathrm{R}(\cdot)$ to form a minimization problem as follows:

$$
\begin{gathered}
\Psi(\boldsymbol{x})=\frac{1}{2}\|\boldsymbol{y}-\boldsymbol{A} \boldsymbol{x}\|^{2}+\mathrm{R}(\boldsymbol{x}) \\
\hat{\boldsymbol{x}}=\arg _{\boldsymbol{x}} \min \Psi(\boldsymbol{x}),
\end{gathered}
$$


where $\|\cdot\|$ is the $\boldsymbol{\ell}_{2}$-norm, and $\|\boldsymbol{y}-\boldsymbol{A x}\|^{2}$ is the data fit term. QPLS can be solved efficiently using iterative algorithms such as conjugate gradient [15]. Although we focus on quadratic regularizers here for ease of analysis, the proposed method can be generalized to regularizers like hyperbola and Huber that are quadratic near zero. However, it is not directly applicable to problems with nonsmooth regularizers, such as $\ell_{1}$ regularizers used for encouraging sparsity in compressed sensing.

Some applications [7]-[9] use a spatial roughness penalty that separately penalizes the real and imaginary parts of $\boldsymbol{x}$ :

$$
\mathrm{R}(\boldsymbol{x})=\frac{1}{2}\left(\beta_{1}\left\|\boldsymbol{C}_{1} \boldsymbol{x}_{\mathrm{R}}\right\|^{2}+\beta_{2}\left\|\boldsymbol{C}_{2} \boldsymbol{x}_{\mathrm{I}}\right\|^{2}\right),
$$

where $\boldsymbol{x}=\boldsymbol{x}_{\mathrm{R}}+\dot{x}_{\mathrm{I}}$ and the subscripts R and I refer to the real and imaginary parts respectively. Also, $\boldsymbol{C}_{1}$ and $\boldsymbol{C}_{2}$ are real-valued matrices that compute finite differences between neighboring voxels of $\boldsymbol{x}_{\mathrm{R}}$ and $\boldsymbol{x}_{\mathrm{I}}$ respectively (most common are first and second order finite differences) and $\beta_{1}$ and $\beta_{2}$ are the regularization parameters that control the tradeoff between noise and spatial resolution in the real $\left(\hat{x_{R}}\right)$ and imaginary $\left(\hat{x}_{\mathrm{I}}\right)$ parts of $\hat{x}$, respectively. We would like to have a fast method for choosing $\beta_{1}$ and $\beta_{2}$ to achieve a desired spatial resolution for $\hat{\boldsymbol{x}}_{\mathrm{R}}$ and $\hat{\boldsymbol{x}_{\mathrm{I}}}$ in (2) based on their local impulse responses.

Before we find their local impulse responses we first consider the special case when $\beta_{1}=\beta_{2}$ $=\beta$ and $\boldsymbol{C}_{1}=\boldsymbol{C}_{2}=\boldsymbol{C}$. In this case, the penalty in (3) simplifies to the conventional roughness penalty as follows:

$$
\mathrm{R}(\boldsymbol{x})=\frac{\beta}{2}\left(\left\|\boldsymbol{C} \boldsymbol{x}_{\mathrm{R}}\right\|^{2}+\left\|\boldsymbol{C} \boldsymbol{x}_{\mathrm{I}}\right\|^{2}\right)=\frac{\beta}{2}\|\boldsymbol{C} \boldsymbol{x}\|^{2} .
$$

Here, it suffices to find the local impulse response of the complex-valued $\hat{\boldsymbol{x}}$. This local impulse response has been derived previously in [10]. The next section reviews this work along with the well-known accelerated way of calculating it using FFTs.

\section{Fast QPLS Local Impulse Response: Conventional Roughness Penalty}

When using (4) in (2), its local impulse response $\boldsymbol{l}_{n}$ at spatial position $n$ is given as follows [10]:

$$
\boldsymbol{l}_{n}=\left(\boldsymbol{A}^{\prime} \boldsymbol{A}+\beta \boldsymbol{C}^{\prime} \boldsymbol{C}\right)^{-1} \boldsymbol{A}^{\prime} \boldsymbol{A} \boldsymbol{e}_{n}, \quad n=1, \ldots, N,
$$

where $\boldsymbol{e}_{n}$ is a vector with 1 at vector element position $n$ and zeros elsewhere (Kronecker impulse).

Evaluating (5) directly to calculate $\boldsymbol{l}_{n}$ would require inverting $\boldsymbol{A}^{\prime} \boldsymbol{A}+\boldsymbol{B} \boldsymbol{C}^{\prime} \boldsymbol{C}$, which could be time consuming and memory intensive. However, if $\boldsymbol{A}{ }^{\prime} \boldsymbol{A}$ and $\boldsymbol{C}^{\prime} \boldsymbol{C}$ are approximately locally 
shift invariant linear transforms, as is often the case, one can use FFT to approximate this inverse rapidly as summarized in the next two subsections.

\section{A. Fast Local Impulse Response Calculation: Circulant Matrices}

Suppose $\boldsymbol{A}{ }^{\prime} \boldsymbol{A}$ and $\boldsymbol{C} \boldsymbol{C}^{\prime}$ are circulant ${ }^{1}$ matrices. Such matrices exhibit two notable properties. First, circulant matrices are diagonalizable using the discrete Fourier transform (DFT), so any linear mapping using $\boldsymbol{A}^{\prime} \boldsymbol{A}$ and $\boldsymbol{C}^{\prime} \boldsymbol{C}$ can be implemented efficiently using the FFT [16]. Second, circulant matrices are shift invariant linear transforms, i.e., a circulant matrix $\boldsymbol{M}$ has the following property:

$$
M e_{n^{\prime}}=S_{n \rightarrow n^{\prime}} M e_{n}, n^{\prime}, n=1, \ldots, N
$$

where $S_{n \rightarrow n^{\prime}}$ is a permutation matrix that circularly shifts the response from position $n$ to position $n^{\prime}$.

The first property allows us to rewrite $\boldsymbol{A}^{\prime} \boldsymbol{A}$ and $\boldsymbol{C}^{\prime} \boldsymbol{C}$ as follows:

$$
A^{\prime} A=Q^{-1} \Lambda Q, \quad C^{\prime} C=Q^{-1} \Omega Q
$$

where $\boldsymbol{Q}$ is the DFT matrix, and $\Lambda$ and $\Omega$ are diagonal matrices given by,

$$
\begin{gathered}
\boldsymbol{\Lambda}=\operatorname{diag}\left\{\boldsymbol{Q} \boldsymbol{A}^{\prime} \boldsymbol{A} \boldsymbol{e}_{1}\right\}, \\
\boldsymbol{\Omega}=\operatorname{diag}\left\{\boldsymbol{Q} \boldsymbol{C}^{\prime} \boldsymbol{C} \boldsymbol{e}_{1}\right\},
\end{gathered}
$$

where $\operatorname{diag}\{\cdot\}$ is a diagonal matrix with its diagonal elements given by its vector argument elements. The second property allows us to find $\Lambda$ and $\Omega$ in (8) for any $\boldsymbol{e}_{n}$ as follows:

$$
\begin{gathered}
\boldsymbol{\Lambda}=\operatorname{diag}\left\{\mathrm{e}^{-i \iota\left(\boldsymbol{Q} \boldsymbol{e}_{n}\right)} \odot\left(\boldsymbol{Q} \boldsymbol{A}^{\prime} \boldsymbol{A} \boldsymbol{e}_{n}\right)\right\}, \\
\boldsymbol{\Omega}=\operatorname{diag}\left\{\mathrm{e}^{-i \iota\left(\boldsymbol{Q} \boldsymbol{e}_{n}\right)} \odot\left(\boldsymbol{Q} \boldsymbol{C}^{\prime} \boldsymbol{C} \boldsymbol{e}_{n}\right)\right\},
\end{gathered}
$$

where $\odot$ is the Hadamard (or element-wise) product, and $\mathrm{e}^{-i \angle\left(\boldsymbol{Q} e_{n}\right)}$ is due to the DFT shift theorem.

Using (7) the local impulse response in (5) simplifies to:

${ }^{1}$ For simplicity we use "circulant" to refer to the general circulant properties of a linear transform of images of any dimension, e.g., in 2D "circulant" refers to "block circulant circulant blocks" (BCCB). 


$$
\boldsymbol{l}_{n}=\left(\boldsymbol{A}^{\prime} \boldsymbol{A}+\beta \boldsymbol{C}^{\prime} \boldsymbol{C}\right)^{-1} \boldsymbol{A}^{\prime} \boldsymbol{A} \boldsymbol{e}_{n},=\boldsymbol{Q}^{-1}(\boldsymbol{\Lambda}+\beta \boldsymbol{\Omega})^{-1} \boldsymbol{\Lambda} \boldsymbol{Q} \boldsymbol{e}_{n},
$$

with $\Lambda$ and $\Omega$ given by (9). Thus when $\boldsymbol{A} \boldsymbol{A}^{\boldsymbol{A}}$ and $\boldsymbol{C}^{\prime} \boldsymbol{C}$ are circulant, one can efficiently calculate the local impulse response using FFT and its inverse (IFFT) for $\boldsymbol{Q}$ and $\boldsymbol{Q}^{-1}$ respectively, and by computing $(\Lambda+\beta \Omega)^{-1} \Lambda$ elementwise.

\section{B. Fast Local Impulse Response Calculation: Approximate Local Shift Invariance}

For most imaging problems $\boldsymbol{A}{ }^{\prime} \boldsymbol{A}$ and $\boldsymbol{C}^{\prime} \boldsymbol{C}$ are not circulant. However, in many cases they are approximately locally shift invariant transforms and thus exhibit similar properties in the neighborhood of any image voxel as a circulant transform would. An approximately locally shift invariant linear transform $\boldsymbol{M}$ has the following property:

$$
M e_{n^{\prime}} \approx S_{n \rightarrow n^{\prime}} M e_{n}, \forall n \in \mathscr{N}_{n^{\prime}}, n^{\prime}=1, \ldots, N
$$

where $\mathscr{N}_{n^{\prime}}$ denotes a neighborhood around voxel index $n^{\prime}$. An example of such a linear transform is when $\boldsymbol{M}$ is Toeplitz ${ }^{2}$. The property described in (11) allows one to approximate $\boldsymbol{M}$ with a circulant based DFT diagonalization as described in Section III-A.

Suppose both $\boldsymbol{C}^{\prime} \boldsymbol{C}$ and $\boldsymbol{A}^{\prime} \boldsymbol{A}$ are approximately locally shift invariant. Using DFT diagonalization and (11), one can locally approximate $\boldsymbol{A}^{\prime} \boldsymbol{A}$ and $\boldsymbol{C}^{\prime} \boldsymbol{C}$ as follows:

$$
A^{\prime} A e_{n} \approx Q^{-1} \check{\Lambda} Q e_{n}, C^{\prime} C e_{n} \approx Q^{-1} \check{\Omega} Q e_{n}
$$

where $\check{\Lambda}$ and $\check{\Omega}$ are defined as given in (9) with an additional constraint that the elements of $\check{\Lambda}$ and $\check{\Omega}$ are nonnegative real. This constraint ensures positive semidefiniteness when locally approximating the positive semidefinate matrices $\boldsymbol{A} \boldsymbol{A}^{\boldsymbol{A}}$ and $\boldsymbol{C}^{\prime} \boldsymbol{C}$.

Using (12) one can approximate the local impulse response in (10) such that:

$$
\boldsymbol{l}_{n} \approx \boldsymbol{Q}^{-1}(\check{\Lambda}+\beta \check{\Omega})^{-1} \check{\Lambda} Q \boldsymbol{e}_{n} .
$$

For most practical penalties, the null space of $\boldsymbol{C}$ is only uniform images, which is disjoint from the null space of $\boldsymbol{A}$. This property, combined with $\check{\Lambda}$ and $\check{\Omega}$ having nonnegative real diagonal elements, makes $\check{\Lambda}+\beta \check{\Omega}$ invertible and thus (13) exists by design.

\footnotetext{
${ }^{2}$ For simplicity we use "Toeplitz" to refer to the general Toeplitz properties of a linear transform of images of any dimension, e.g., in 2D "Toeplitz" referes to "block Toeplitz Toeplitz blocks" (BTTB).
} 


\section{Fast QPLS Local Impulse Response: Seperate Real and Imaginary Roughness Penalty}

The method used to derive the local impulse response presented in Section III does not apply directly when using the more complicated penalty in (3). To analyze the local impulse response when using (3), we form a stacked cost function with separate real and imaginary regularization. This stacked cost function looks like $\Psi(x)$ with the conventional penalty in (4) and thus allows us to derive a local impulse response of the stacked cost function similar to (5), with all the computational benefits presented in Section III-B.

\section{A. Stacked Cost Function With Separate Real and Imaginary Regularization}

We first rewrite all the matrices and vectors in (2) and (3) in a stacked format, as follows:

$$
\begin{gathered}
\boldsymbol{y}_{\mathrm{S}}=\left[\begin{array}{l}
\boldsymbol{y}_{\mathrm{R}} \\
\boldsymbol{y}_{\mathrm{I}}
\end{array}\right], \quad \boldsymbol{x}_{\mathrm{S}}=\left[\begin{array}{c}
\boldsymbol{x}_{\mathrm{R}} \\
\boldsymbol{x}_{\mathrm{I}}
\end{array}\right], \\
\boldsymbol{A}_{\mathrm{S}}=\left[\begin{array}{cc}
\boldsymbol{A}_{\mathrm{R}} & -\boldsymbol{A}_{\mathrm{I}} \\
\boldsymbol{A}_{\mathrm{I}} & \boldsymbol{A}_{\mathrm{R}}
\end{array}\right], \quad \boldsymbol{C}_{\mathrm{S}}=\left[\begin{array}{cc}
\boldsymbol{C}_{\mathrm{S} 1} & 0 \\
0 & \boldsymbol{C}_{\mathrm{S} 2}
\end{array}\right],
\end{gathered}
$$

where $\boldsymbol{C}_{\mathrm{S}}$ is block diagonal with $\boldsymbol{C}_{\mathrm{S} 1}=\sqrt{\beta_{1}} C_{1}$ and $\boldsymbol{C}_{\mathrm{S} 2}=\sqrt{\beta_{2}} C_{2}$. Note that $\beta_{1}$ and $\beta_{2}$ can be chosen independently of each other and this flexibility is needed in some applications [7][9]. Using these definitions for the stacked matrices and vectors we form a new stacked cost function $\Psi_{\mathrm{S}}\left(\boldsymbol{x}_{\mathrm{S}}\right)$ as follows:

$$
\Psi_{\mathrm{S}}\left(\boldsymbol{x}_{\mathrm{S}}\right)=\frac{1}{2}\left\|\boldsymbol{y}_{\mathrm{S}}-\boldsymbol{A}_{\mathrm{S}} \boldsymbol{x}_{\mathrm{S}}\right\|^{2}+\frac{1}{2}\left\|\boldsymbol{C}_{\mathrm{S}} \boldsymbol{x}_{\mathrm{S}}\right\|^{2}
$$

The Appendix shows that by minimizing $\Psi_{\mathrm{S}}\left(\boldsymbol{x}_{\mathrm{S}}\right)$ with respect to $\boldsymbol{x}_{\mathrm{S}}$ we are regularizing the real and imaginary parts separately and maintaining equality with $\Psi(\boldsymbol{x})$. Note that we use (14) for resolution analysis only, not for numerical implementation of the image reconstruction method. Next we characterize the spatial resolution properties of (14) in term of its stacked local impulse response.

\section{B. Stacked Local Impulse Response}

Since the stacked cost function in (14) has the same general form as the QPLS cost function using the conventional penalty in (4), we can use the method in [10] to derive the stacked local impulse response. It is thus written as follows:

$$
\boldsymbol{l}_{\mathrm{S}_{n}}=\left(A_{\mathrm{S}}^{\prime} A_{\mathrm{S}}+C_{\mathrm{S}}^{\prime} C_{\mathrm{S}}\right)^{-1} A_{\mathrm{S}}^{\prime} A_{\mathrm{S}} e_{\mathrm{S}_{n}}
$$

with, 


$$
\begin{gathered}
\boldsymbol{l}_{\mathrm{S}_{n}}=\left[\begin{array}{c}
\boldsymbol{l}_{\mathrm{R}_{n}} \\
\boldsymbol{l}_{\mathrm{I}_{n}}
\end{array}\right], \quad \boldsymbol{e}_{\mathrm{S}_{n}}=\left[\begin{array}{c}
(1-\alpha) \boldsymbol{e}_{n} \\
\alpha \boldsymbol{e}_{n}
\end{array}\right], \quad \alpha \in\{0,1\} \\
\boldsymbol{A}_{\mathrm{S}}^{\prime} \boldsymbol{A}_{\mathrm{S}}=\left[\begin{array}{cc}
\Re\left(\boldsymbol{A}^{\prime} \boldsymbol{A}\right) & -\mathfrak{J}\left(\boldsymbol{A}^{\prime} \boldsymbol{A}\right) \\
\mathfrak{J}\left(\boldsymbol{A}^{\prime} \boldsymbol{A}\right) & \Re\left(\boldsymbol{A}^{\prime} \boldsymbol{A}\right)
\end{array}\right] \\
\boldsymbol{C}_{\mathrm{S}}^{\prime} \boldsymbol{C}_{\mathrm{S}}=\left[\begin{array}{cc}
\beta_{1} \boldsymbol{C}_{1}^{\prime} \boldsymbol{C}_{1} & \mathbf{0} \\
\mathbf{0} & \beta_{2} \boldsymbol{C}_{2}^{\prime} \boldsymbol{C}_{2}
\end{array}\right],
\end{gathered}
$$

where $\Re\left(\boldsymbol{A}^{\prime} \boldsymbol{A}\right)$ and $\Im\left(\boldsymbol{A}^{\prime} \boldsymbol{A}\right)$ are the real and imaginary parts of $\boldsymbol{A}^{\prime} \boldsymbol{A}$ respectively, and $\boldsymbol{a}$ is set to 0 or 1 to calculate the stacked local impulse response of $\boldsymbol{x}_{\mathrm{R}}$ or $\boldsymbol{x}_{\mathrm{I}}$ respectively. Using (15) we could in principle characterize the spatial properties of a QPLS reconstruction that separately penalizes the real and imaginary parts. However, as is the case with (5), calculating $\boldsymbol{l}_{\mathbf{S} n}$ directly would be both computationally and memory intensive. By using a procedure akin to that in Section III-B we accelerate this computation using FFTs.

\section{Fast Stacked Local Impulse Response Calculation: Approximate Local Shift Invariance}

Suppose $\boldsymbol{A}^{\prime} \boldsymbol{A}, \boldsymbol{C}_{1}^{\prime} \boldsymbol{C}_{1}$ and $\boldsymbol{C}_{2}^{\prime} \boldsymbol{C}_{2}$ are approximately locally shift invariant. DFT diagonalization and (11) then gives:

$$
\begin{array}{r}
\Re\left(\boldsymbol{A}^{\prime} \boldsymbol{A}\right) \boldsymbol{e}_{n} \approx \boldsymbol{Q}^{-1} \check{\boldsymbol{\Lambda}}_{1} \boldsymbol{Q} \boldsymbol{e}_{n}, \\
\Im\left(\boldsymbol{A}^{\prime} \boldsymbol{A}\right) \boldsymbol{e}_{n} \approx \boldsymbol{Q}^{-1} \check{\boldsymbol{\Lambda}}_{2} \boldsymbol{Q} \boldsymbol{e}_{n}, \\
\boldsymbol{C}_{1}^{\prime} \boldsymbol{C}_{1} \boldsymbol{e}_{n} \approx \boldsymbol{Q}^{-1} \check{\boldsymbol{\Omega}}_{1} \boldsymbol{Q} \boldsymbol{e}_{n}, \\
\boldsymbol{C}_{2}^{\prime} \boldsymbol{C}_{2} \boldsymbol{e}_{n} \approx \boldsymbol{Q}^{-1} \check{\boldsymbol{\Omega}}_{2} Q \boldsymbol{e}_{n},
\end{array}
$$

where $\check{\Lambda}=\check{\Lambda_{1}}+i \check{\Lambda_{2}}$. The diagonal matrices $\check{\Lambda}, \check{\Omega}_{1}$ and $\check{\Omega}_{2}$ are defined similar to (9) with the additional constraint of having only nonnegative real elements. Substituting (17) into (15) allows us to approximate the stacked local impulse response as follows:

$$
\boldsymbol{l}_{\mathrm{S}_{n}} \approx \boldsymbol{Q}_{\mathrm{S}}^{-1}\left(\check{\Lambda}_{\mathrm{S}}+\check{\Omega}_{\mathrm{S}}\right)^{-1} \check{\Lambda}_{\mathrm{S}} \boldsymbol{Q}_{\mathrm{S}} \boldsymbol{e}_{\mathrm{S}_{n}}
$$


with

$$
\begin{gathered}
\check{\boldsymbol{\Lambda}}_{\mathrm{S}}=\left[\begin{array}{cc}
\check{\boldsymbol{\Lambda}}_{1} & -\check{\check{\Lambda}}_{2} \\
\check{\boldsymbol{\Lambda}}_{2} & \check{\boldsymbol{\Lambda}}_{1}
\end{array}\right], \quad \check{\boldsymbol{\Omega}}_{\mathrm{S}}=\left[\begin{array}{cc}
\beta_{1} \check{\Omega}_{1} & \mathbf{0} \\
\mathbf{0} & \beta_{2} \check{\Omega}_{2}
\end{array}\right], \\
\boldsymbol{Q}_{\mathrm{S}}=\left[\begin{array}{cc}
\boldsymbol{Q} & \mathbf{0} \\
\mathbf{0} & \boldsymbol{Q}
\end{array}\right] .
\end{gathered}
$$

The expression in (18) is getting closer to being practical for fast computation using FFTs, but we still must address the fact that $\check{\Lambda_{\mathrm{S}}}$ is not a diagonal matrix.

To compute (18) in a stable manner, $\check{\Lambda_{\mathrm{S}}}+\check{\Omega}_{\mathrm{S}}$ must be invertible for all positive $\beta_{1}$ and $\beta_{2}$. For this to be true, it suffices to show that (a) the eigenvalues of $\check{\Lambda_{\mathrm{S}}}$ and $\check{\Omega}_{\mathrm{S}}$ are nonnegative real, and (b) $\boldsymbol{A}_{\mathrm{S}}$ and $\boldsymbol{C}_{\mathrm{S}}$ have disjoint null spaces.

For (b), we note that by converting each vector of the complex-valued null space basis of $\boldsymbol{A}$ to its stacked format, they form a basis of the null space of $\boldsymbol{A}_{\mathrm{S}}$. The same relationship holds for the null space basis vectors of $\boldsymbol{C}$ and $\boldsymbol{C}_{\mathrm{S}}$. Hence, since $\boldsymbol{C}$ and $\boldsymbol{A}$ have disjoint null spaces by design, statement (b) above is true.

For (a), we note that since $\check{\Omega}_{\mathrm{S}}$ is a diagonal matrix with nonnegative real diagonal elements according to (19), it suffices to show that the eigenvalues of $\overline{\Lambda_{\mathrm{S}}}$ are nonnegative real. To show this, we need to express the eigenvalues of $\check{\Lambda_{\mathrm{S}}}$ in terms of the nonnegative real diagonal elements of $\check{\Lambda}$.

First, we need to express the elements of $\check{\Lambda_{1}}$ and $\check{\Lambda_{2}}$ as functions of the elements of $\check{\Lambda}$. Using the approximation in (12), we get the following:

$\boldsymbol{Q R}\left(\boldsymbol{A}^{\prime} \boldsymbol{A}\right) \boldsymbol{e}_{n}=Q \Re\left(\boldsymbol{A}^{\prime} \boldsymbol{A} \boldsymbol{e}_{n}\right) \approx Q \Re\left(\boldsymbol{Q}^{-1} \check{\boldsymbol{\Lambda}} \boldsymbol{Q} \boldsymbol{e}_{n}\right)=Q \frac{Q^{-1} \check{\Lambda} Q \boldsymbol{e}_{n}+\left(\boldsymbol{Q}^{-1} \check{\Lambda} Q e_{n}\right)^{*}}{2}=\frac{1}{2} \boldsymbol{v}+\frac{1}{2} \boldsymbol{Q}\left(\boldsymbol{Q}^{-1} \boldsymbol{v}\right)^{*}$,

where * is the conjugate operator and $v$ is a vector of length $K$ with its $k$ th element given as:

$$
v_{k}=\check{\lambda}_{k} \mathrm{e}^{i<\left(\boldsymbol{Q} \boldsymbol{e}_{n}\right)_{k},}
$$

where $\check{\lambda_{k}}$ is the $k$ th diagonal element of $\check{\Lambda}$. The expression in (20) involves a conjugate of the IDFT of $v$, which for element $n$ in the vector simplifies as follows: 


$$
\left[\left(\boldsymbol{Q}^{-1} \boldsymbol{v}\right)^{*}\right]_{n}=\left(\sum_{k=0}^{K-1} Q_{\mathrm{nk}} v_{k}\right)^{*}=\sum_{k=0}^{K-1} Q_{\mathrm{nk}}^{*} v_{k}^{*}=\sum_{k=0}^{K-1} Q_{\mathrm{nk}} v_{\underline{k}}^{*}=\left[\boldsymbol{Q}^{-1} \underline{\boldsymbol{v}}^{*}\right]_{n}
$$

where $\underline{k}$ is the conjugate index that goes with $k$ and $\underline{v}$ has those elements, e.g., in $1 \mathrm{D}$ its $k$ th element is defined as follows:

$$
[\underline{\boldsymbol{v}}]_{k}=v_{\underline{k}}= \begin{cases}v_{0} & \text { if } k=0, \\ v_{K-k} & \text { if } k=1, \ldots, K-1 .\end{cases}
$$

Thus $\left(\boldsymbol{Q}^{-1} \boldsymbol{v}\right)^{*}$ is simply an IDFT of $\underline{\boldsymbol{v}}^{*}$. Using this in (20), we now get for the $k$ th element:

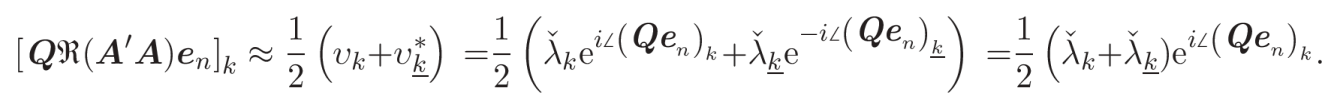

Using this expression and (17), the diagonal elements of $\check{\Lambda_{1}}$, denoted $\check{\lambda_{1}}$, relate to the nonnegative real elements of $\check{\Lambda}$ as follows:

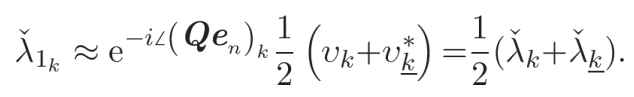

Thus the elements of $\check{\Lambda_{1}}$ are approximately nonnegative real under the local impulse response approximation. Similarly, the elements of $\check{\Lambda_{2}}$ under the local impulse response approximation relate to the elements of $\check{\Lambda}$ as follows:

$$
\check{\lambda}_{2_{k}} \approx \frac{1}{2 i}\left(\check{\lambda}_{k}-\check{\lambda}_{\underline{k}}\right)
$$

making the elements of $\check{\Lambda_{2}}$ approximately purely imaginary.

The matrix $\check{\Lambda_{\mathrm{S}}}$ in (19) is a $2 \times 2$ block matrix where each block is a $K \times K$ diagonal matrix and the main diagonal is approximately nonnegative real and the off-diagonals are approximately imaginary. Although this form does not tell us yet if the eigenvalues of $\check{\Lambda_{\mathrm{S}}}$ are nonnegative real, we can use elementary row and column matrix operations to permute it into a block diagonal matrix and determine if the eigenvalues of each block are nonnegative real. If this is the case, then the eigenvalues of $\check{\mathrm{S}}$ are also nonnegative real since its eigenvalues are invariant to elementary matrix operations.

Permuting $\check{\Lambda_{\mathrm{S}}}$ into a block diagonal matrix, it has $K$ blocks of size $2 \times 2$, with the $k$ th one given as follows: 


$$
\left[\begin{array}{cc}
\check{\lambda}_{1_{k}} & -\check{\lambda}_{2_{k}} \\
\check{\lambda}_{2_{k}} & \check{\lambda}_{1_{k}}
\end{array}\right] .
$$

Since all the blocks along the diagonal have the same form, we need only determine the eigenvalues of (23). We find the eigenvalues by solving the following characteristic equation:

$$
\begin{aligned}
& \left|\begin{array}{cc}
\check{\lambda}_{1_{k}}-\alpha & -\check{\lambda}_{2_{k}} \\
\check{\lambda}_{2_{k}} & \check{\lambda}_{1_{k}}-\alpha
\end{array}\right|=0 \\
& \Rightarrow \alpha^{2}-2 \check{\lambda}_{1_{k}} \alpha+\check{\lambda}_{1_{k}}^{2}+\check{\lambda}_{2_{k}}^{2}=0 .
\end{aligned}
$$

Using (21) and (22) the two roots $a_{1,2}$ are:

$$
\begin{aligned}
& \alpha_{1,2}=\frac{2 \check{\lambda}_{1_{k}} \pm \sqrt{4 \check{\lambda}_{1_{k}}^{2}-4\left(\check{\lambda}_{1_{k}}^{2}+\check{\lambda}_{2_{k}}^{2}\right)}}{2} \\
& =\frac{2 \check{\lambda}_{1_{k}} \pm \sqrt{-4 \check{\lambda}_{2_{k}}^{2}} \approx \frac{1}{2}\left(\check{\lambda}_{k}+\check{\lambda}_{\underline{k}}\right) \pm \frac{1}{2} \sqrt{\left(\check{\lambda}_{k}-\check{\lambda}_{\underline{k}}\right)^{2}}}{2} \\
& =\frac{1}{2}\left(\check{\lambda}_{k}+\check{\lambda}_{\underline{k}}\right) \pm \frac{1}{2}\left(\check{\lambda}_{k}-\check{\lambda}_{\underline{k}}\right) \\
& =\left\{\check{\lambda}_{k}, \check{\lambda}_{\underline{k}}\right\} .
\end{aligned}
$$

Since all elements of $\check{\Lambda}$ have been constrained to be nonnegative real under the local impulse response approximation, $a_{1,2}$, and hence the eigenvalues of $\check{\Lambda_{\mathrm{S}}}$, are also nonnegative real.

By enforcing the approximation in (21) and (22) and by constraining the elements of $\check{\Lambda}$ to be nonnegative real, we have now shown that $\check{\Lambda}_{\mathrm{S}}$ is positive semidefinite. This property, in conjunction with $\check{\Omega}_{\mathrm{S}}$ being positive semidefinite, and $\boldsymbol{A}_{\mathrm{S}}$ and $\boldsymbol{C}_{\mathrm{S}}$ having disjoint null spaces, ensures that $\check{\Lambda_{\mathrm{S}}}+\check{\Omega}_{\mathrm{S}}$ is invertible. Thus the proposed approximate stacked local impulse response in (18) exists and can be evaluated quickly.

\section{Fast Stacked Local Impulse Response Calculation: Implementation Details}

To implement a fast version of the stacked local impulse response approximation in (18), we need to form $\check{\Lambda}_{\mathrm{S}}$ and $\check{\Omega}_{\mathrm{S}}$ fast and efficiently. To do that we use (9) to form $\check{\check{\Lambda}_{\bar{\Omega}}} \check{\Omega}_{1}$ and $\check{\Omega}_{2}$ and constrain their diagonals to be nonnegative real by discarding the imaginary part and setting the real part to be 0 if it is negative. We then use (21) and (22) to form $\check{\Lambda_{1}}$ and $\check{\Lambda_{2}}$ respectively. We compute $\left(\check{\Lambda_{\mathrm{S}}}+\check{\Omega}_{\mathrm{S}}\right)^{-1}$ quickly by using the $2 \times 2$ block matrix structure of $\check{\Lambda_{\mathrm{S}}}$ and $\check{\Omega}_{\mathrm{S}}$ shown in (19) and a blockwise matrix inversion. Since all the blocks are diagonal matrices this inversion is trivial to calculate. As usual we use FFT and IFFT for $\boldsymbol{Q}$ and $\boldsymbol{Q}^{-1}$ respectively. 


\section{Simulations \& Results}

To evaluate the accuracy of the fast local impulse responses proposed in (13) and (18), we investigated the resolution properties of two MR image reconstruction methods, and compared the result of (13) and (18) to that of using their respective slower, but exact, versions in (5) and (15). Both image reconstruction methods are used for functional MRI (fMRI). The first is an off-resonance corrected $T_{2}^{*}$-weighted image reconstruction method with the conventional roughness penalty [17], and the second is a joint $R_{2}^{*}$ and field map image reconstruction method with a separate real $\left(R_{2}^{*}\right)$ and imaginary (field map) roughness penalty [8].

The off-resonance corrected $T_{2}^{*}$-weighted image reconstruction method with the conventional roughness penalty is given as follows [17]:

$$
\begin{aligned}
& \hat{\boldsymbol{x}}=\underset{\boldsymbol{x}}{\arg \min } \frac{1}{2}\|\boldsymbol{y}-\boldsymbol{A} \boldsymbol{x}\|^{2}+\frac{\beta}{2}\|\boldsymbol{C} \boldsymbol{x}\|^{2}, \\
& a_{\mathrm{mn}}=\Phi\left(\vec{k}\left(t_{m}\right)\right) \mathrm{e}^{-\mathrm{it}_{m} \omega\left(\vec{r}_{n}\right)} \mathrm{e}^{-i 2 \pi\left(\vec{k}\left(t_{m}\right) \cdot \vec{r}_{n}\right)},
\end{aligned}
$$

where $\hat{\boldsymbol{x}}$ is the estimated $T_{2}^{*}$-weighted image, $\boldsymbol{C}$ is a first order difference matrix, and $a_{m n}$ is an element of the $M \times N$ system matrix $\boldsymbol{A}$ for voxel index $n$ and k-space trajectory index $m$. For $a_{m n}, \vec{r}_{n}$ is the spatial voxel location, $\vec{k}\left(t_{m}\right)$ is the k-space trajectory value, $\Phi\left(\vec{k}\left(t_{m}\right)\right)$ is the Fourier transform value of the continuous-to-discrete basis function, and $\omega\left(\vec{r}_{n}\right)$ is the field map value used for voxel-based off-resonance correction. The joint $R_{2}^{*}$ and field map image reconstruction with the separate real and imaginary roughness penalty is given as follows [8]:

$$
\begin{gathered}
\hat{\boldsymbol{x}}=\underset{\boldsymbol{x}}{\arg \min } \frac{1}{2}\|\boldsymbol{y}-\boldsymbol{A} \boldsymbol{x}\|^{2}+\frac{\beta_{1}}{2}\left\|\boldsymbol{C}_{1} \boldsymbol{x}_{\mathrm{R}}\right\|^{2}+\frac{\beta_{2}}{2}\left\|\boldsymbol{C}_{2} \boldsymbol{x}_{\mathrm{I}}\right\|^{2}, \\
a_{\mathrm{mn}}=\Phi\left(\vec{k}\left(t_{m}\right)\right) f\left(\vec{r}_{n}\right) \mathrm{e}^{-t_{m} \check{x}\left(\vec{r}_{n}\right)}\left(-t_{m}\right) \mathrm{e}^{-i 2 \pi\left(\vec{k}\left(t_{m}\right) \cdot \vec{r}_{n}\right)},
\end{gathered}
$$

where $\hat{\boldsymbol{x}}=\hat{\boldsymbol{x}}_{\mathrm{R}}+\dot{\boldsymbol{x}} \hat{\mathrm{I}}$ is the jointly estimated $R_{2}^{*}\left(\hat{\boldsymbol{x}}_{\mathrm{R}}\right)$ and field map $\left(\hat{\boldsymbol{x}}_{\mathrm{I}}\right)$ images, $\boldsymbol{C}_{1}$ is a first order difference matrix, $\boldsymbol{C}_{2}$ is a second order difference matrix (per [18]), and $a_{m n}$ is a system matrix element that has, in addition to the variables in (24), $f\left(\vec{r}_{n}\right)$ as the objects initial magnetization voxel value after RF excitation, and the complex valued $\check{x}\left(\vec{\Gamma}_{n}\right)$ as a reference $R_{2}^{*}$ (real part) and field map (imaginary part) voxel value that is assumed to be close to $x\left(\vec{I}_{n}\right)$ to be estimated.

While most variables that make $a_{m n}$ in (24) and (25) are known, spatial maps of $\omega\left(\vec{r}_{n}\right), f\left(\vec{r}_{n}\right)$, and $\check{x}\left(\vec{r}_{n}\right)$ are usually estimated from additionally acquired data. Here, we will assume they 
are known a priori for all $n$. To investigate the performance of the proposed fast local impulse response method, we simulated six versions of $\boldsymbol{A}$ in (24) and (25) by using three commonly used k-space trajectories $\vec{k}(t)$ in fMRI, and two sets of the spatial simulation maps $\boldsymbol{\omega}, \boldsymbol{f}$, and $\check{\boldsymbol{x}}$.

The three single-shot $\vec{k}(t)$ trajectories used for the simulations (TE $=30 \mathrm{~ms}, \mathrm{FOV}=220 \times$ $220 \mathrm{~mm}, 4 \mu \mathrm{s} \mathrm{sampling}$ ) were spiral-out, spiral-in, and echo-planar imaging (EPI), each respectively with a readout time of $18.8 \mathrm{~ms}, 18.8 \mathrm{~ms}$, and $24.2 \mathrm{~ms}$, and sample count $M$ of 4713,4713 , and 4096. For the two sets of $64 \times 64$-sized simulation maps $(N=4096)$, the first set simulated a simple case, where $f$ was set to a constant value inside a simulated brain and 0 outside it, and $\boldsymbol{\omega}$ and $\check{\boldsymbol{x}}$ were both set to 0 . While $\boldsymbol{A}^{\prime} \boldsymbol{A}$ is generally not Toeplitz for both (24) and (25), it is for this simple set of simulation maps and hence is well suited to the approximate local shift invariance property needed for accurate fast local impulse response calculation. Fig. 1 shows the second set of simulation maps, based on a previously acquired in vivo human brain fMRI images and thus more realistic. As is common in many reconstruction problems, $\boldsymbol{A}^{\prime} \boldsymbol{A}$ is now approximately Toeplitz, since most voxels in Fig. 1 have a smooth spatial transition to neighboring voxels, except voxels by the ventricle and brain edges.

To investigate the resolution properties of (24) and (25), the simulations required multiple evaluations of the fast and slow local impulse responses, while recording their FWHM dependence on either the regularization parameter values $\left(\beta, \beta_{1}\right.$, or $\left.\beta_{2}\right)$ or the spatial location of the Kronecker impulse $\left(\boldsymbol{e}_{n}\right)$. Both local impulse response methods were computed and timed in MATLAB R2015b on a workstation with two 6-core Intel Xeon E5-2630 2.3GHz CPUs and 64GB of memory. We made the slow local impulse response computationally efficient, by either precomputing $\boldsymbol{A}^{\prime} \boldsymbol{A}$ when evaluating the FWHM dependence on the regularization parameters, or $\left(\boldsymbol{A}^{\prime} \boldsymbol{A}+\boldsymbol{\beta} \boldsymbol{C}^{\prime} \boldsymbol{C}\right)^{-1} \boldsymbol{A}^{\prime} \boldsymbol{A}$ (or its stacked version) when evaluating the FWHM dependence on the Kronecker impulse location. To make the fast local impulse response computationally efficient when evaluating its FWHM dependence on the regularization parameters we precomputed $\boldsymbol{A}^{\prime} \boldsymbol{A} \boldsymbol{e}_{n}$, while no precomputation was performed when evaluating the FWHM dependence on the Kronecker impulse locations. Furthermore, the fast method formed $\boldsymbol{A}$ and $\boldsymbol{C}$ in a memory efficient manner ${ }^{3}$ [8], [17], [19].

\section{A. Comparing FWHM Values of The Slow and Fast Local Impulse Response Methods at a Single Spatial Location}

For both the slow and fast local impulse response methods, we calculated the FWHM from the magnitude of the complexvalued local impulse response of the center voxel across a range of regularization parameter values. The range was chosen to result in reasonable FWHM values for (24) and (25).

Fig. 2 shows plots of the resulting FWHM dependence on $\beta$ for the slow and fast local impulse responses of (24). This is shown for the three k-space trajectories, and the simple and human brain simulation maps. Since the system matrix in (24) requires only a field map

${ }^{3}$ Available from http://www.eecs.umich.edu/ fessler/code 
out of the simulation maps, Fig. 2a shows the FWHM results for the simple (all zeros) field map and Fig. 2b shows it for the human brain field map in Fig. 1c.

To further quantify the difference between the FWHM of the fast and slow impulse response methods shown in Fig. 2, Fig. 3 shows a plot of the FWHM\%-Error across the same range of $\beta$ values. Here, $\mathrm{FWHM} \%$-Error is defined as follows,

$$
\mathrm{FWHM}_{\%-\text { Error }}=\frac{\mathrm{FWHM}_{\text {fast }}-\mathrm{FWHM}_{\text {slow }}}{\mathrm{FWHM}_{\text {slow }}} \cdot 100 \% \text {, }
$$

where $\mathrm{FWHM}_{\text {fast }}$ and $\mathrm{FWHM}_{\text {slow }}$ are respectively the FWHM of the fast and slow local impulse responses. Fig. 3 shows that $\mathrm{FWHM}_{\text {fast }}$ is slightly smaller than $\mathrm{FWHM}_{\text {slow }}$ across the chosen $\beta$ range, indicating that the fast method tends to underestimate the resolution. The average absolute FWHM\%-Error for the spiral-out, spiral-in, and EPI k-space trajectories were less than $1.2 \%$ and $3.7 \%$ for the simple and human brain field maps respectively. This shows that the FWHM values with the simple (all zeros) field map has a lower average absolute FWHM\%-Error compared to when the human brain field map is used. This is most likely due to $\boldsymbol{A}^{\prime} \boldsymbol{A}$ being Toeplitz in (24) when the field map is all zeros.

When using (25) to jointly reconstruct $R_{2}^{*}$ and field map, we can adjust two regularization parameter values, $\beta_{1}$ and $\beta_{2}$. For every pair of $\beta_{1}$ and $\beta_{2}$ values, we get separate estimates of the complex-valued local impulse responses for $R_{2}^{*}$ and field map by respectively setting $a$ to 0 or 1 in (15) for the slow method and (18) for the fast method. For each estimate we calculate the $R_{2}^{*} \mathrm{FWHM}\left(\mathrm{FWHM}_{\mathrm{R}}\right)$ and field map FWHM $\left(\mathrm{FWHM}_{\mathrm{I}}\right)$ from the magnitude of their complex-valued local impulse responses. From this we form two FWHM maps as functions of $\beta_{1}$ and $\beta_{2}$, i.e., $\operatorname{FWHM}_{\mathrm{R}}\left(\beta_{1}, \beta_{2}\right)$ and $\operatorname{FWHM}_{\mathrm{I}}\left(\beta_{1}, \beta_{2}\right)$.

Fig. 4 shows profiles of the resulting $\mathrm{FWHM}_{\mathrm{R}}$ and $\mathrm{FWHM}_{\mathrm{I}}$ maps, for the three k-space trajectories, and the simple and human brain simulation maps. The profiles were selected by fixing $\beta_{1}$ at $\hat{\beta}_{1}$ or $\beta_{2}$ at $\hat{\beta}_{2}$ and plot $\operatorname{FWHM}_{\mathrm{R}}\left(\beta_{1}, \hat{\beta}_{2}\right)$ and $\operatorname{FWHM}_{\mathrm{I}}\left(\hat{\beta}_{1}, \beta_{2}\right)$. Here, we chose $\hat{\beta}_{1}$ and $\hat{\beta}_{2}$ as follows,

$$
\begin{aligned}
& \hat{\beta}_{1}=\underset{\beta_{1} \in \mathbb{B}}{\arg \min }\left(\left(\frac{1}{N_{\beta_{2}}} \sum_{\beta_{2}} \operatorname{FWHM}_{\mathrm{R}}\left(\beta_{1}, \beta_{2}\right)\right)-1.35\right), \\
& \hat{\beta}_{2}=\underset{\beta_{2} \in \mathbb{B}}{\arg \min }\left(\left(\frac{1}{N_{\beta_{1}}} \sum_{\beta_{1}} \operatorname{FWHM}_{\mathrm{I}}\left(\beta_{1}, \beta_{2}\right)\right)-1.7\right),
\end{aligned}
$$

where $\mathrm{B}$ is the set of $\beta_{1}$ and $\beta_{2}$ values used to generate Fig. 4 , and $N_{\beta_{1}}=N_{\beta_{2}}=17$ is the number of $\beta_{1}$ and $\beta_{2}$ values for a total of $289\left(\beta_{1}, \beta_{2}\right)$ pairs. Here, we chose 1.35 and 1.7 as sensible FWHM targets for $R_{2}^{*}$ and field map respectively. 
Fig. $4 a-4 b$ show the $\mathrm{FWHM}_{\mathrm{R}}$ and $\mathrm{FWHM}_{\mathrm{I}}$ profiles for the simple simulation maps, and Fig. 4c-4d for the human brain simulation maps. Similar to Fig. 2, Fig. 4 shows a good agreement between the $\mathrm{FWHM}_{\mathrm{R}}$ and $\mathrm{FWHM}_{\mathrm{I}}$ of the slow and fast local impulse responses. This is further highlighted in Fig. 5 where the $\mathrm{FWHM}_{\mathrm{R}}$ and $\mathrm{FWHM}_{\mathrm{I}}$ percent errors are shown in Fig. 5a and 5b respectively. Averaged across $\beta_{1}$ or $\beta_{2}$, the absolute valued $R_{2}^{*}$ and field map FWHM\%-Error for the three k-space trajectories are all within $2.2 \%$ for both sets of simulation maps. Again we see from Fig. 5 that for the chosen profiles the fast method largely underestimated the FWHM values compared to the slow method, although less so for higher $\beta_{1}$ and $\beta_{2}$ values. When evaluating the results across all the $289\left(\beta_{1}, \beta_{2}\right)$ pairs, the average absolute valued $R_{2}^{*}$ and field map FWHM\%-Error was less than $3.1 \%$ in all cases.

We averaged the time for computing the FWHM values for all the regularization parameter values used for the conventional regularizer (24), with equal regularization of the two parts, and separate regularization (25) across the six combinations of k-space trajectories and simulation maps. The slow method took 35.8s (5.2s to precompute $\boldsymbol{A}^{\prime} \boldsymbol{A}$ and 30.6 s to compute the local impulse response for eleven $\beta \mathrm{s}$ ) for (24), and $1289.5 \mathrm{~s}$ (10.5s to precompute $\boldsymbol{A}^{\prime} \boldsymbol{A}$ and 1279.0 s to compute the real and imaginary local impulse responses for all $\left(\beta_{1}, \beta_{2}\right)$ pairs) for (25). The fast method took 0.8s (0.75s to precompute $\boldsymbol{A}^{\prime} \boldsymbol{A} \boldsymbol{e}_{n}$ and $0.04 \mathrm{~s}$ to compute the local impulse responses for eleven $\beta \mathrm{s}$ ) for (24), and $4.5 \mathrm{~s}$ ( $2.4 \mathrm{~s}$ to precompute $\boldsymbol{A}^{\prime} \boldsymbol{A} \boldsymbol{e}_{n}$ and 2.1s to compute both real and imaginary local impulse response for all $\left(\beta_{1}, \beta_{2}\right)$ pairs) for (25). The fast method thus provided on average approximately 44-fold and 286fold acceleration for (24) and (25) respectively.

\section{B. FWHM Spatial Mapping for Spatially Varying Regularization}

Section V-A demonstrated how the proposed method can quickly and accurately tabulate the $\mathrm{FWHM}_{\mathrm{R}}$ and $\mathrm{FWHM}_{\mathrm{I}}$ of the local impulse responses at a single voxel location, by sweeping through a range of regularization parameters. From these tables we can then estimate the $\beta_{1}$ and $\beta_{2}$ values to produce a predetermined image resolution. This is done by selecting desired FWHM values for the real and imaginary parts based on practical experience with fMRI, e.g., here we used 1.35 and 1.7 pixel FWHM for the real and imaginary parts respectively, since the field map in the imaginary part is smooth. Then we invert (using a simple 2D interpolation) the FWHM tables to find the corresponding $\beta_{1}$ and $\beta_{2}$ values that provide the desired spatial resolution. For the simple case when $\beta_{1}$ equals $\beta_{2}$, the inversion simplifies to a 1D interpolation. Fig. $2 \& 4$ show how this inversion is trivial since the FWHMs are monotone increasing functions of $\beta$ s.

Since the FWHM tables are formed for a single voxel location, it may in some cases not reflect accurately the resolution properties at other spatial locations. This is especially relevant when $\boldsymbol{A}^{\prime} \boldsymbol{A}$ for the image reconstruction is not shift invariant or nearly so, e.g., circulant or Toeplitz. In that case, and depending on the purpose of the image reconstruction, it can be important to investigate the local spatial resolution properties at multiple voxel locations and incorporate such information into the regularization [10]. The now spatially dependent estimated regularization parameter values can then be integrated into the penalty in the form of a weighting map $\boldsymbol{x}=\left[1, \ldots, \kappa_{n}, \ldots, \kappa_{N}\right]^{T}$, where $\kappa_{n}$ is the estimated 
regularization parameter at spatial index $n[10]$. Furthermore, the separate real and imaginary penalty requires two such spatial weighting maps, $\boldsymbol{x}_{R}$ and $\boldsymbol{\kappa}_{I}$ respectively.

To estimate $\boldsymbol{\kappa}$ for (24), and $\boldsymbol{\kappa}_{R}$ and $\boldsymbol{\kappa}_{I}$ for (25), we used the fast and slow local impulse response methods. For both (24) and (25) we only used the human brain simulation maps in Fig. 1 to demonstrate a more realistic example of spatial variation of the local impulse response due to $\boldsymbol{A}^{\prime} \boldsymbol{A}$ not being shift invariant. This was explored for all three k-space trajectories. For 942 internal brain voxels (the brain edge voxels were excluded), we tabulated the FWHM of the local impulse responses across the same range of $\beta, \beta_{1}$, and $\beta_{2}$ values used in Section V-A. For the $T_{2}^{*}$-weighted reconstruction in (24) the average compute time across all voxels for the fast method was $6.6 \mathrm{~s}\left(0.6 \mathrm{~s}\right.$ per $\beta$ ), and for the joint $R_{2}^{*}$ and field map reconstruction in (25) it was $194.1 \mathrm{~s}\left(0.7 \mathrm{~s}\right.$ per $\left(\beta_{1}, \beta_{2}\right)$ pair). The same computation using the slow method took respectively $97.2 \mathrm{~s}$ and 7180 s, which is respectively almost 15 and 37-times slower.

From the resulting FWHM tabulation of the proposed fast method, we estimated $\beta, \beta_{1}$, and $\beta_{2}$ at the 942 voxel locations to respectively give a FWHM, FWHM $\mathrm{R}$ and $\mathrm{FWHM}_{\mathrm{I}}$ of 1.35 , 1.35 and 1.7 as described earlier. The regularizers in (24) and (25) used these voxel dependent estimates as $\boldsymbol{\kappa}, \boldsymbol{\kappa}_{R}$, and $\boldsymbol{\kappa}_{I}$ maps. The voxels of the $\boldsymbol{\kappa}, \boldsymbol{\kappa}_{R}$, and $\boldsymbol{\kappa}_{I}$ maps that were outside the 942 voxels were set to the spatial average of their respectively estimated $\beta, \beta_{1}$, and $\beta_{2}$ values. These averages were also used to calculate normalized $\boldsymbol{x}, \boldsymbol{x}_{R}$, and $\boldsymbol{x}_{I}$ maps, respectively named $\check{\boldsymbol{\kappa}}, \check{\boldsymbol{\kappa}}_{R}$, and $\check{\kappa}_{\boldsymbol{I}}$.

Fig. 6 shows a $3 \times 3$ image matrix of the resulting $\check{\boldsymbol{x}}_{,} \check{\boldsymbol{x}}_{R}$, and $\check{\boldsymbol{\kappa}}_{I}$ maps. The rows of the image matrix show the results across the penalized image reconstructions and the columns shows them across k-space trajectories. We note that both image reconstructions require higher regularization parameter values in areas of high intensity in the simulation field map in Fig. 1c, although this is less prominent for the spiral-in k-space trajectory. There is also a notable difference in the overall smoothness between $\check{\boldsymbol{\kappa}}$ (top row) and either $\check{\boldsymbol{\kappa}}_{R}$ or $\check{\boldsymbol{\kappa}}_{I}$ (bottom two rows). This is due to $f$ being in the system matrix in (25), causing a spatial structure that affects the separate real and imaginary roughness penalty.

We used the $\boldsymbol{\kappa}, \boldsymbol{x}_{R}$, and $\boldsymbol{\kappa}_{I}$ maps to form spatially varying roughness penalties [10] for both (24) and (25). We then computed, for the 942 voxel locations, the fast local impulse response for (24) and (25) with said spatially varying roughness penalties and formed a map of their FWHM values. This process was repeated for (24) and (25) while using spatially invariant penalties, where their regularization parameters were estimated from the FWHM tables of the central image voxel. The resulting FWHM maps from both processes were compared to assess the spatial resolution difference between using spatially variant and invariant penalties.

Fig. 7 shows the resulting FWHM spatial maps for the $T_{2}^{*}$-weighted (Fig. 7a), and joint $R_{2}^{*}$ (Fig. 7b) and field map (Fig. 7c) reconstructions. The image matrix rows of Fig. 7a-7c show the results when using the spatially variant (top row) and invariant (bottom row) roughness penalties and the columns show them for different k-space trajectories. Each color scale for Fig. $7 \mathrm{a}-7 \mathrm{c}$ has the desired FWHM as the central value. The FWHM variation across the 
brain when using the spatially invariant penalties (bottom row) can be significant, particularly in areas where Fig. 1c is high, while when using the spatially variant penalties (top row) the FWHM values are much closer to the desired FWHM across the whole brain.

To further asses the accuracy of the fast methods we generated the FWHM maps with the slow methods using the same process. Out of the six combinations of k-space trajectories and simulation maps, the joint reconstruction (spiralin, field map estimate) gave the poorest average, median, and interquartile range for the absolute $\mathrm{FWHM}_{\% \text {-Error }}$ across all the brain voxels $(5.8 \%, 4.2 \%$, and $6.9 \%$ respectively).

\section{Discussion}

We presented a fast method to compute an approximate local impulse response for QPLS image reconstructions (2) with a quadratic roughness penalty that separately penalizes the real and imaginary parts (3). This method was shown to be stable, fast and accurate by comparing the performance of the fast and slow methods for a joint $R_{2}^{*}$ and field map image reconstruction of fMRI data. The fast method was shown to have an absolute FWHM\%-Error average value within $3.1 \%$ for a range of regularization parameter values and 286-times faster than the slow method. Similarly, when using the conventional roughness penalty in (4) (a special case of (3)) for a $T_{2}^{*}$-weighted image reconstruction of fMRI data, the fast method was also shown to be stable, fast and accurate, with an absolute FWHM\%-Error average value less than $3.7 \%$ and 44 -times faster.

We also presented an example of reduced spatial resolution uniformity when using a spatially invariant roughness penalty for a QPLS image reconstruction with shift variant resolution properties, an unfortunate side-effect of some QPLS image reconstructions. By designing an appropriate spatially variant roughness penalty [10] using the fast local impulse response method, we were able to improve spatial resolution uniformity considerably. Furthermore, when using the fast method for an image reconstruction with either a separate real and imaginary or conventional roughness penalty, the average, median, and interquartile

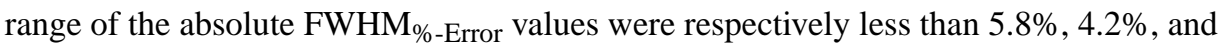
$6.9 \%$. It was also 37 -or 15-times faster than the slow method for the two penalties.

All our simulations were based on reconstructing $64 \times 64$ images. Although it is a modest size, it is still the most common one for fMRI. Recently, fMRI imaging has been moving towards acquiring higher resolution 3D images [20]-[23], resulting in increased compute time and memory needs for the slow methods compared to the fast methods, potentially leading to further speed advantages for the fast methods.

Our analysis was strictly focused on quadratic roughness penalties and is easily extended to other regularizers that are locally quadratic near zero. Furthermore, there is prior work where regularization modulation methods designed for quadratic regularizers as described here were adapted to nonquadratic regularizers [24], as was recently shown for an edge preserving image reconstruction for quantitative susceptibility mapping (QSM) [25]. Extending this work to non-smooth regularizers like $\ell_{1}$ is a challenging open problem. 
Finally, analysis of other separate penalties is also needed, such as separate magnitude and phase penalties [4], [6], [26].

\section{Acknowledgments}

This work was supported by NIH P01 CA087634, and NIH R01 EB023618.

\section{Appendix}

\section{Comparing the Minimization of $\Psi_{\mathrm{S}}\left(x_{\mathrm{S}}\right)$ and $\Psi(x)$}

This appendix verifies that the stacked minimization problem in (14) is equivalent to the original problem in (2).

To show that $\left\|\boldsymbol{y}_{\mathrm{S}}-\boldsymbol{A}_{\mathrm{S}} \boldsymbol{x}_{\mathrm{S}}\right\|^{2}=\|\boldsymbol{y}-\boldsymbol{A} \boldsymbol{x}\|^{2}$ we first note the following relationship:

$$
\boldsymbol{y}_{\mathrm{S}}-\boldsymbol{A}_{\mathrm{S}} \boldsymbol{x}_{\mathrm{S}}=\left[\begin{array}{c}
\boldsymbol{y}_{\mathrm{R}} \\
\boldsymbol{y}_{\mathrm{I}}
\end{array}\right]-\left[\begin{array}{cc}
\boldsymbol{A}_{\mathrm{R}} & -\boldsymbol{A}_{\mathrm{I}} \\
\boldsymbol{A}_{\mathrm{I}} & \boldsymbol{A}_{\mathrm{R}}
\end{array}\right]\left[\begin{array}{c}
\boldsymbol{x}_{\mathrm{R}} \\
\boldsymbol{x}_{\mathrm{I}}
\end{array}\right]=\left[\begin{array}{c}
\mathfrak{R}(\boldsymbol{y}-\boldsymbol{A} \boldsymbol{x}) \\
\mathfrak{I}(\boldsymbol{y}-\boldsymbol{A} \boldsymbol{x})
\end{array}\right] \text {. }
$$

From this we see that the error of the stacked vectors equals the stacked error of the original complex valued vectors. This allows us to relate the two norms as follows:

$$
\left\|\boldsymbol{y}_{\mathrm{S}}-\boldsymbol{A}_{\mathrm{S}} \boldsymbol{x}_{\mathrm{S}}\right\|^{2}=\left\|\left[\begin{array}{c}
\mathfrak{R}(\boldsymbol{y}-\boldsymbol{A} \boldsymbol{x}) \\
\mathfrak{I}(\boldsymbol{y}-\boldsymbol{A} \boldsymbol{x})
\end{array}\right]\right\|^{2}=\|\boldsymbol{y}-\boldsymbol{A} \boldsymbol{x}\|^{2},
$$

and hence the data fit terms of $\Psi(\boldsymbol{x})$ and $\Psi_{\mathrm{S}}\left(\boldsymbol{x}_{\mathrm{S}}\right)$ are equivalent.

It is easy to show that the stacked penalty in (14) is equivalent to the penalty in (3), as follows:

$$
\frac{1}{2}\left\|\boldsymbol{C}_{\mathrm{S}} \boldsymbol{x}_{\mathrm{S}}\right\|^{2}=\frac{1}{2} \boldsymbol{x}_{\mathrm{S}}^{\prime} \boldsymbol{C}_{\mathrm{S}}^{\prime} \boldsymbol{C}_{\mathrm{S}} \boldsymbol{x}_{\mathrm{S}}=\frac{1}{2}\left(\beta_{1} \boldsymbol{x}_{\mathrm{R}}^{\prime} \boldsymbol{C}_{1}^{\prime} \boldsymbol{C}_{1} \boldsymbol{x}_{\mathrm{R}}+\beta_{2} \boldsymbol{x}_{\mathrm{I}}^{\prime} \boldsymbol{C}_{2}^{\prime} \boldsymbol{C}_{2} \boldsymbol{x}_{\mathrm{I}}\right)=\frac{1}{2}\left(\beta_{1}\left\|\boldsymbol{C}_{1} \boldsymbol{x}_{\mathrm{R}}\right\|^{2}+\beta_{2}\left\|\boldsymbol{C}_{2} \boldsymbol{x}_{\mathrm{I}}\right\|^{2}\right)
$$

\section{References}

1. Lange K. Convergence of EM image reconstruction algorithms with Gibbs smoothing. IEEE Trans. Med. Imag. Dec.1990 9(4):439-46.

2. Wang, Q., Zenge, M., Cetingul, HE., Mueller, E., Nadar, MS. Novel Sampling Strategies for Sparse MR Image Reconstruction. Proceedings of the 22nd ISMRM; Conference Proceedings; 2014. p. 1549

3. Chiew M, Smith SM, Koopmans PJ, Graedel NN, Blumensath T, Miller KL. k-t FASTER: Acceleration of functional MRI data acquisition using low rank constraints. Magn Reson Med. 2015; 74(2):353-364. [PubMed: 25168207]

4. Fessler, JA., Noll, DC. Iterative image reconstruction in MRI with separate magnitude and phase regularization. Biomedical Imaging: Nano to Macro, 2004. IEEE International Symposium on, vol. 2. IEEE; Conference Proceedings; 2004. p. 209-12. 
5. Çetin M, Karl WC. Feature-enhanced synthetic aperture radar image formation based on nonquadratic regularization. IEEE Trans. Image Process. Apr.2001 10(4):623-31. [PubMed: 18249651]

6. Zhao F, Noll DC, Nielsen JF, Fessler JA. Separate magnitude and phase regularization via compressed sensing. IEEE Trans. Med. Imag. Feb.2012 31(9):1713-23.

7. Sotthivirat S, Fessler JA. Penalized-likelihood image reconstruction for digital holography. JOSA A. May; 2004 21(5):737-50. [PubMed: 15139426]

8. Olafsson VT, Noll DC, Fessler JA. Fast joint reconstruction of dynamic R2* and field maps in functional MRI. IEEE Trans. Med. Imag. Sep.2008 27(9):1177-88.

9. Bydder M, Robson MD. Partial Fourier partially parallel imaging. Magn Reson Med. Jun.2005 53(6):1393-401. [PubMed: 15906299]

10. Fessler JA, Rogers WL. Spatial resolution properties of penalized-likelihood image reconstruction: space-invariant tomographs. IEEE Trans. Image Process. Sep.1996 5(9):1346-58. [PubMed: 18285223]

11. Ramani S, Liu Z, Rosen J, Nielsen JF, Fessler JA. Regularization parameter selection for nonlinear iterative image restoration and MRI reconstruction using GCV and SURE-based methods. IEEE Trans. Image Process. Aug.2012 21(8):3659-72. [PubMed: 22531764]

12. Wilson, DW., Tsui, BMW. Spatial resolution properties of FB and ML-EM reconstruction methods. Nuclear Science Symposium and Medical Imaging Conference, 1993., 1993 IEEE Conference Record; Conference Proceedings; 1993. p. 1189-1193.

13. Qi J, Leahy RM. Resolution and noise properties of MAP reconstruction for fully 3-D PET. IEEE Trans. Med. Imag. May; 2000 19(5):493-506.

14. Fessler JA. Model-Based Image Reconstruction for MRI. IEEE Signal Process Mag. Jul.2010 27(4):81-89. [PubMed: 21135916]

15. Pruessmann KP, Weiger M, Bornert P, Boesiger P. Advances in sensitivity encoding with arbitrary k-space trajectories. Magn Reson Med. 2001; 46(4):638-51. [PubMed: 11590639]

16. Golub, GH., Van Loan, CF. Matrix computations. 3. Baltimore: Johns Hopkins University Press; 1996. ser. Johns Hopkins studies in the mathematical sciences.

17. Sutton BP, Noll DC, Fessler JA. Fast, iterative image reconstruction for MRI in the presence of field inhomogeneities. IEEE Trans. Med. Imag. Feb.2003 22(2):178-88.

18. Funai AK, Fessler JA, Yeo DT, Olafsson VT, Noll DC. Regularized field map estimation in MRI. IEEE Trans. Med. Imag. Oct.2008 27(10):1484-94.

19. Fessler JA, Sutton BP. Nonuniform fast Fourier transforms using min-max interpolation. IEEE Transactions on Signal Processing. Feb.2003 51(2):560-574.

20. Setsompop K, Gagoski BA, Polimeni JR, Witzel T, Wedeen VJ, Wald LL. Blipped-controlled aliasing in parallel imaging for simultaneous multislice echo planar imaging with reduced g-factor penalty. Magn Reson Med. May; 2012 67(5):1210-24. [PubMed: 21858868]

21. Smith SM, Beckmann CF, Andersson J, Auerbach EJ, Bijsterbosch J, Douaud G, Duff E, Feinberg DA, Griffanti L, Harms MP, Kelly M, Laumann T, Miller KL, Moeller S, Petersen S, Power J, Salimi-Khorshidi G, Snyder AZ, Vu AT, Woolrich MW, Xu J, Yacoub E, Ugurbil K, Van Essen DC, Glasser MF, Consortium WU-MH. Resting-state fMRI in the Human Connectome Project. Neuroimage. Oct.2013 80:144-68. [PubMed: 23702415]

22. Posse S, Ackley E, Mutihac R, Rick J, Shane M, Murray-Krezan C, Zaitsev M, Speck O. Enhancement of temporal resolution and BOLD sensitivity in real-time fMRI using multi-slab echo-volumar imaging. Neuroimage. May; 2012 61(1):115-30. [PubMed: 22398395]

23. Olafsson V, Kundu P, Wong EC, Bandettini PA, Liu TT. Enhanced identification of BOLD-like components with multi-echo simultaneous multi-slice (MESMS) fMRI and multi-echo ICA. Neuroimage. May.2015 112:43-51. [PubMed: 25743045]

24. Ahn S, Leahy RM. Analysis of resolution and noise properties of nonquadratically regularized image reconstruction methods for PET. IEEE Trans. Med. Imag. Mar.2008 27(3):413-424.

25. Chun SY. Spatial resolution properties of QSM images using MEDI algorithm. Proceedings of the 25th ISMRM. 2017:1461.

26. Zibetti MVW, De Pierro AR. Improving compressive sensing in MRI with separate magnitude and phase priors. Multidimensional Systems and Signal Processing. 2016:1-23. 


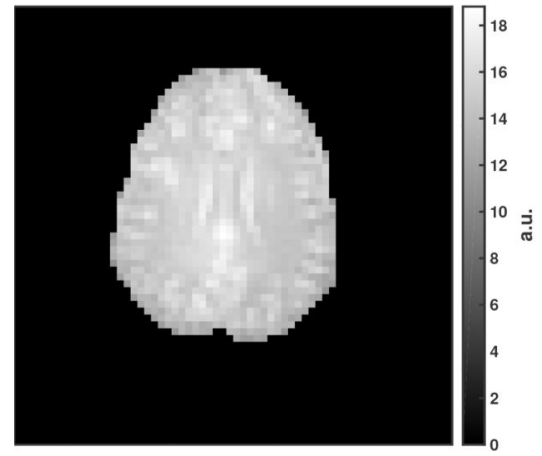

(a)

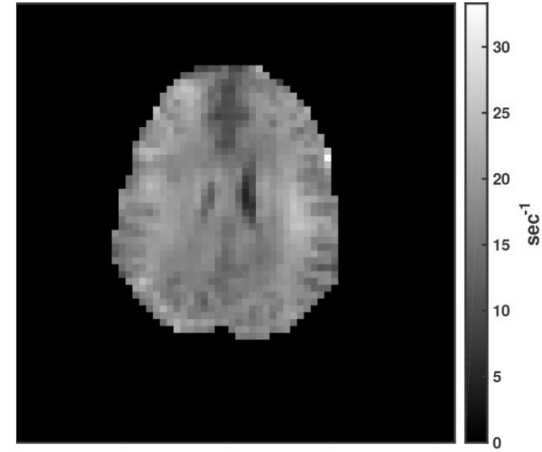

(b)

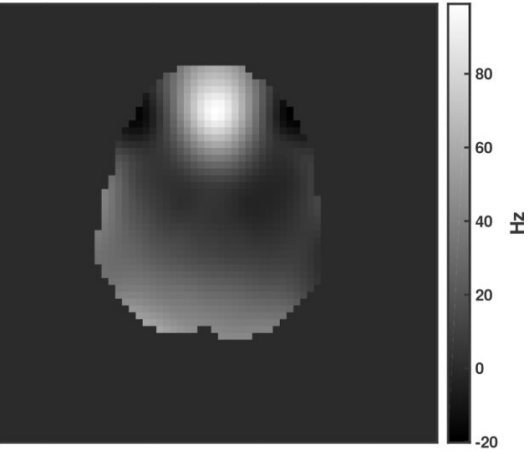

(c)

Fig. 1.

Set of $64 \times 64$ simulation maps, from a previously acquired in vivo human brain fMRI data set, that is used to investigate the performance of the fast local impulse response method. (a) Initial magnetization map $f$; (b) $R_{2}^{*}$ map; (c) Field map $\omega$. 


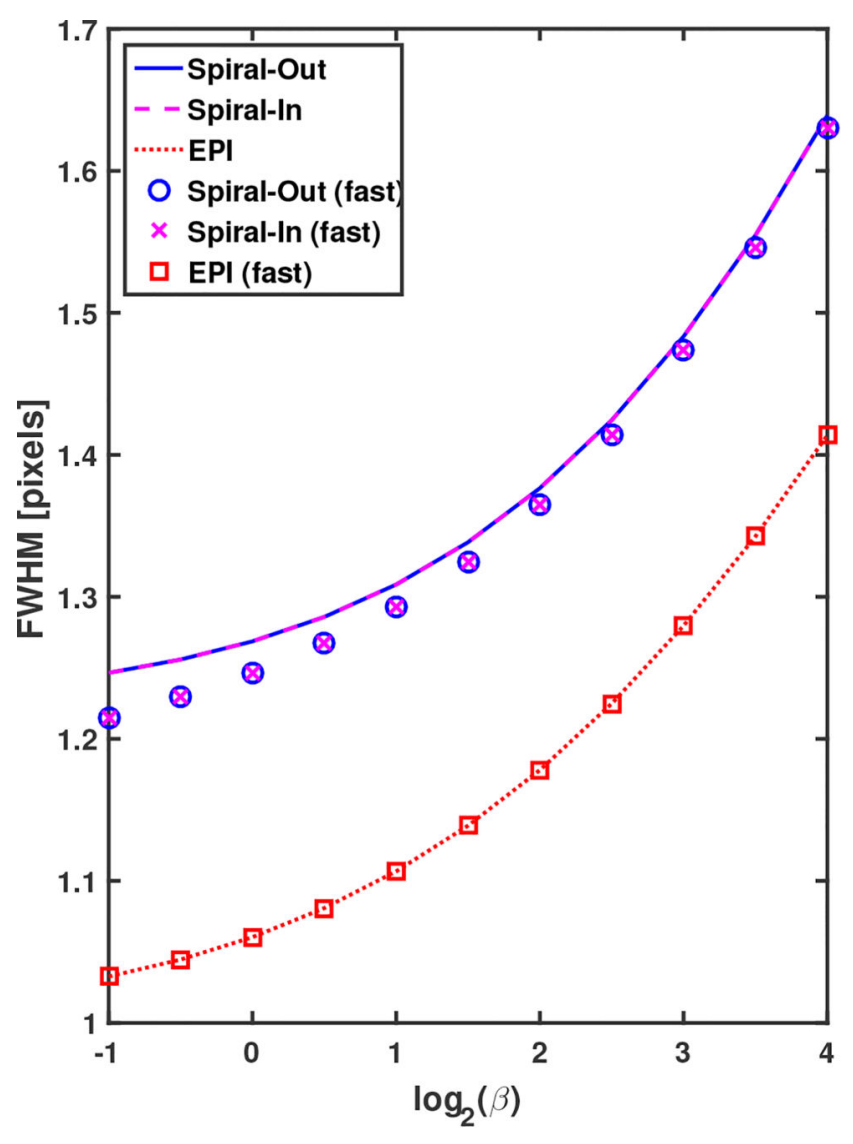

(a)

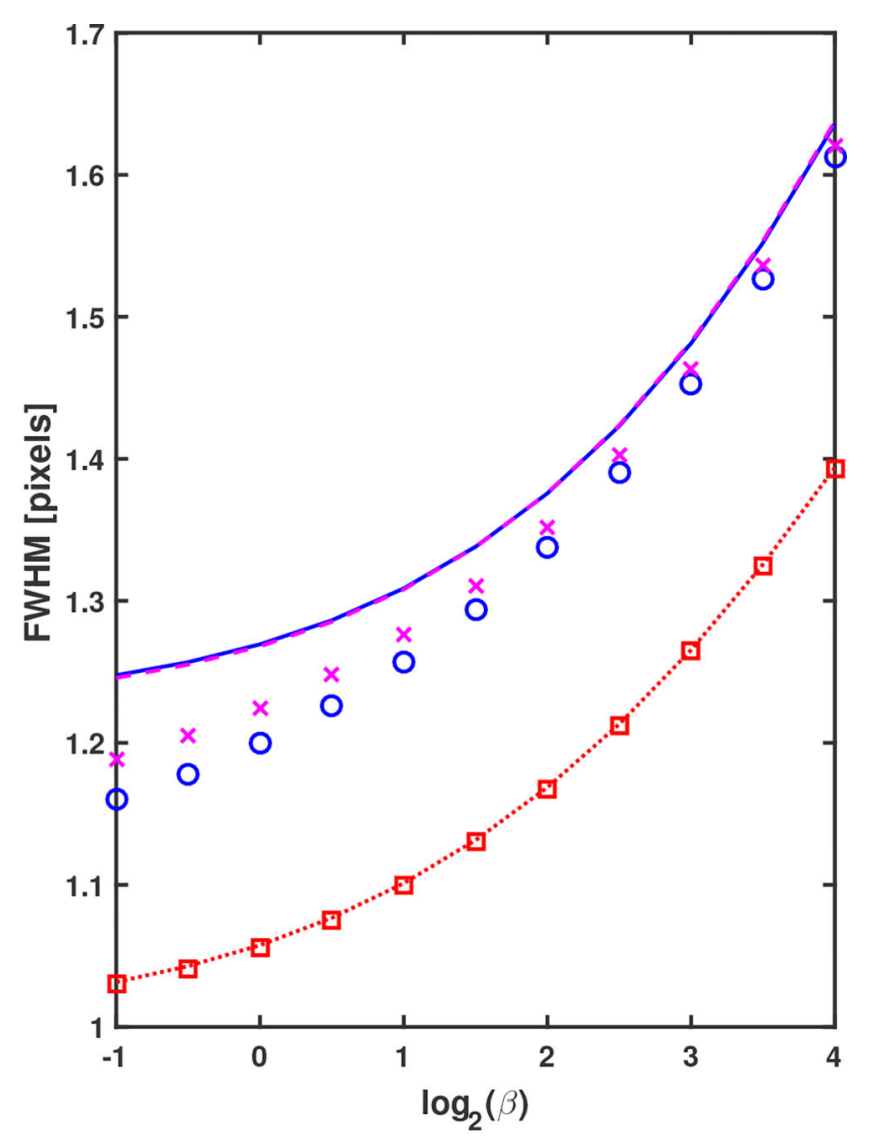

(b)

Fig. 2.

Plots of the FWHM dependence on the regularization parameter $\beta$ for the slow and fast local impulse responses for the $T_{2}^{*}$-weighted image reconstruction in (24). The plots show the FWHM of the local impulse response evaluated at the center image voxel. This was performed for the spiral-out, spiral-in, and EPI k-space trajectories, using both the (a) simple and (b) human brain field maps. 


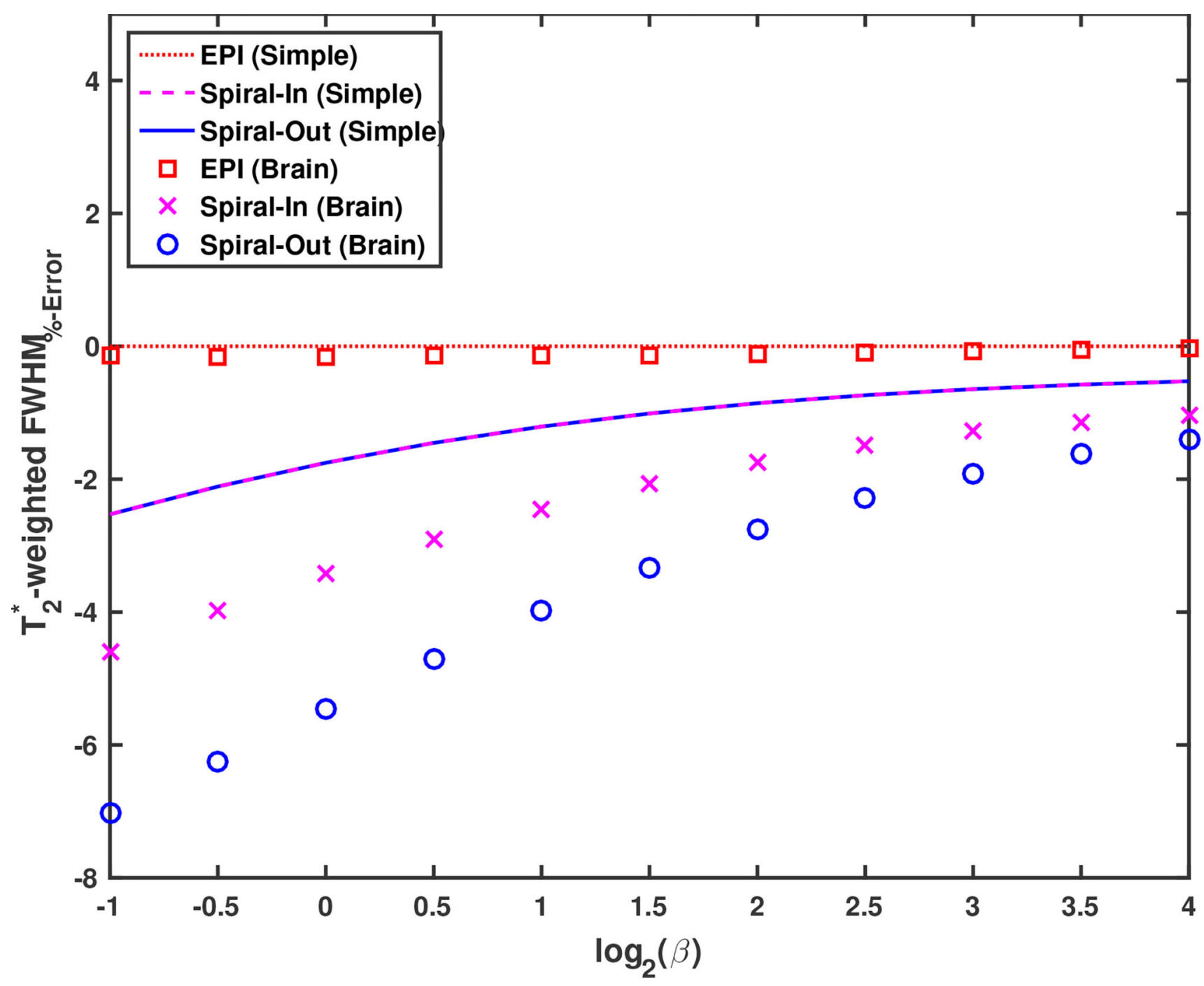

Fig. 3.

Plots of the FWHM $\%$-Error dependence on the regularization parameter $\beta$ for the $T_{2}^{*}$ weighted image reconstruction in (24), for all three k-space trajectories and both sets of simulation field maps. In all case the error is less than $7.1 \%$. 


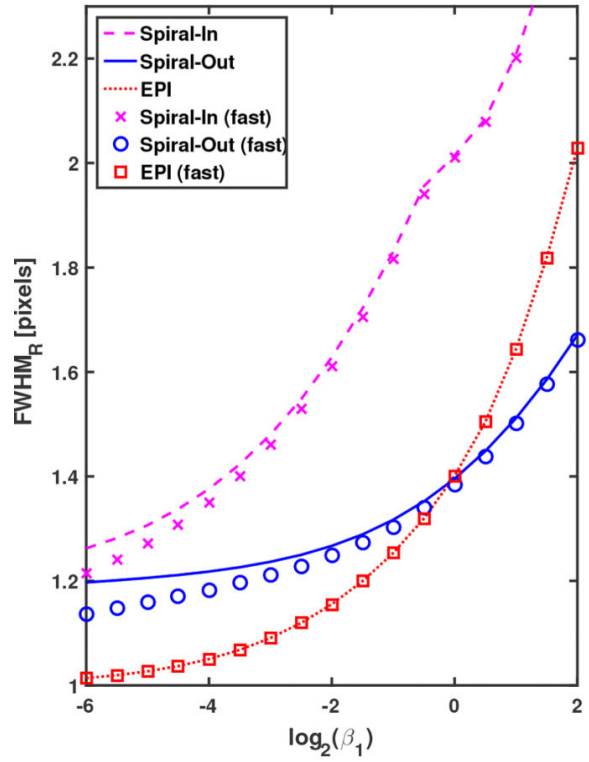

(a)

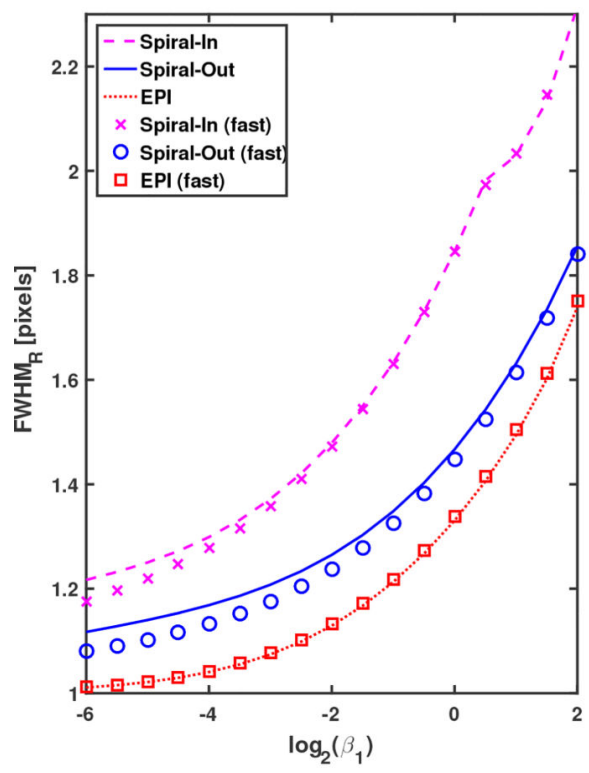

(c)

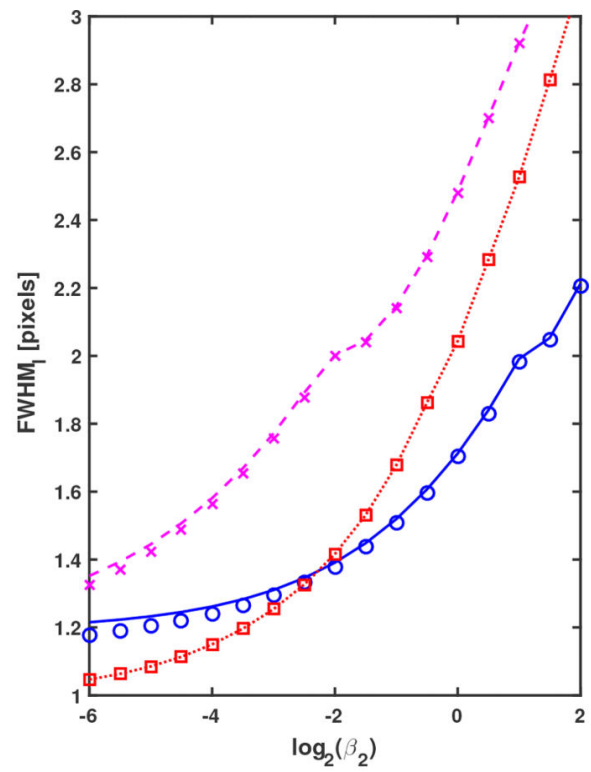

(b)

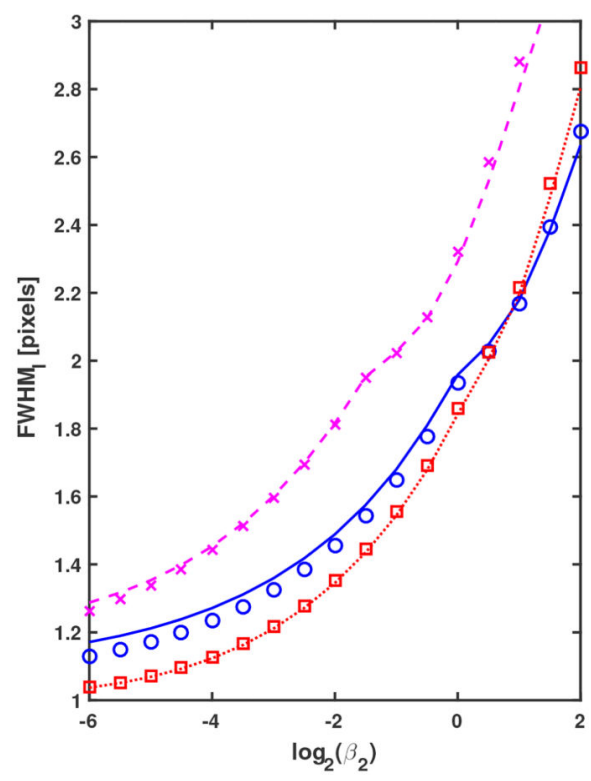

(d)

Fig. 4.

Profiles of the FWHM dependence on the regularization parameter values $\beta_{1}$ and $\beta_{2}$ of the slow and fast local impulse response methods for the joint $R_{2}^{*}$ and field map image reconstruction in (25). The results for the $R_{2}^{*}$ FWHM with the spiral-in, spiral-out, and EPI $\mathrm{k}$-space trajectories are shown for the (a) simple and (c) human brain simulation maps. Similarly, the field map FWHM are shown for the (b) simple and (d) human brain simulation maps. 


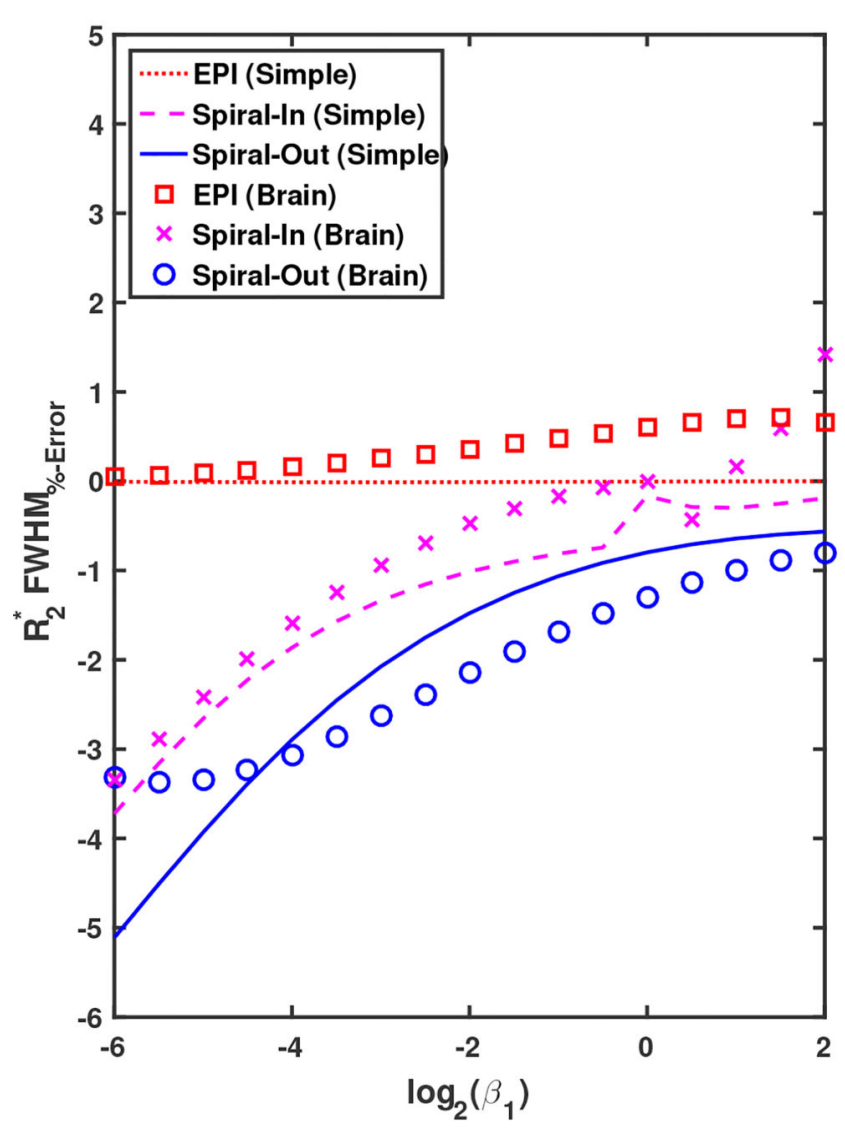

(a)

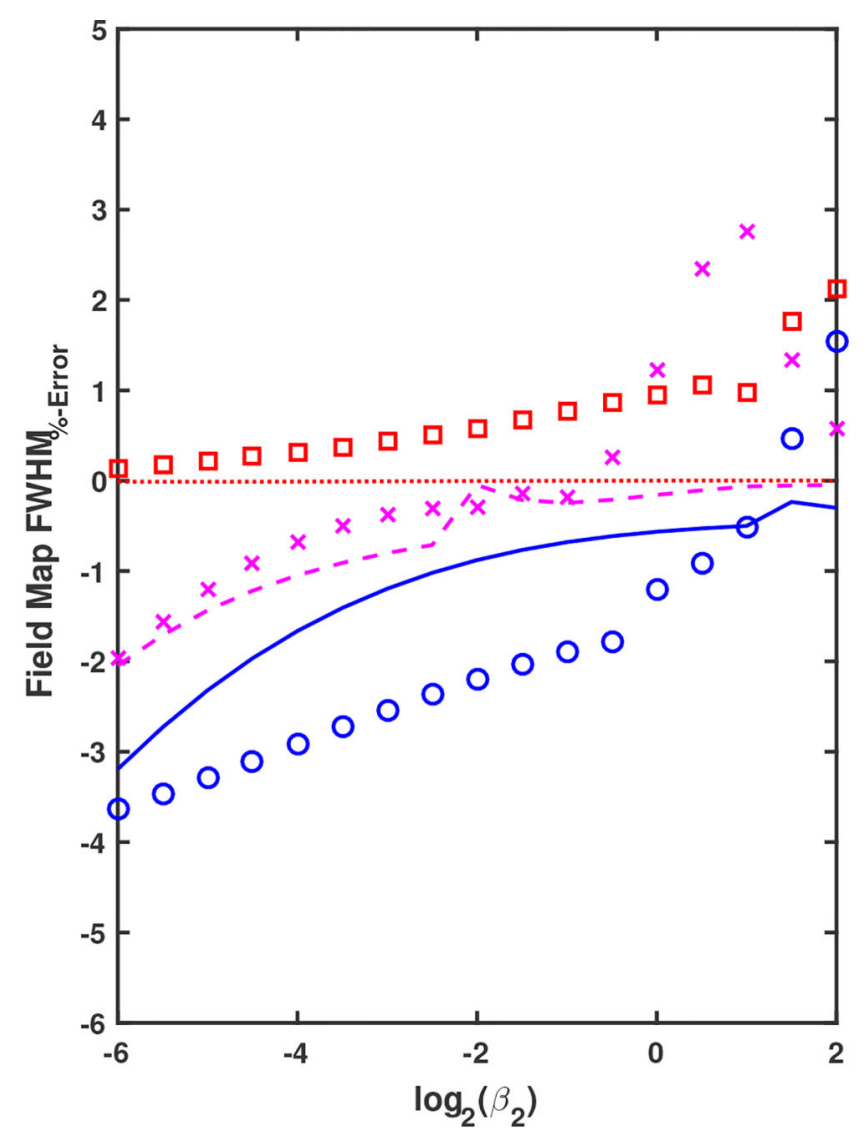

(b)

Fig. 5.

Plots of the $R_{2}^{*}$ and field map FWHM $\%$-Error dependence on the $\beta_{1}$ and $\beta_{2}$ values for the profiles in Fig. 4. This is shown for all three k-space trajectories and both sets of simulation maps for (a) $R_{2}^{*}$ and (b) field map. The absolute error for each $\beta_{1}$ or $\beta_{2}$ profile value is less than $5.2 \%$ and averaged across $\beta_{1}$ or $\beta_{2}$ is less than $2.2 \%$. 


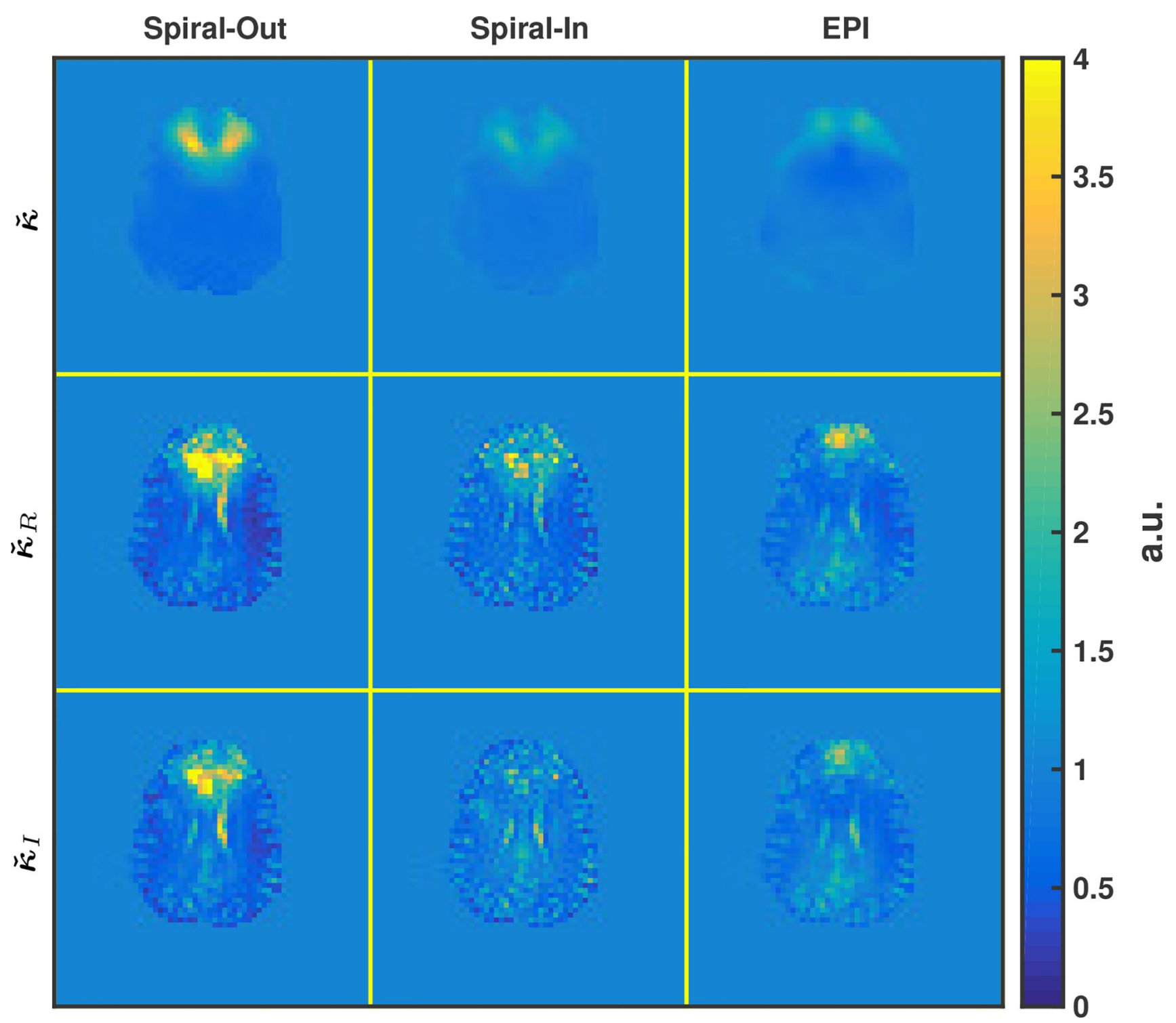

Fig. 6 .

The $3 \times 3$ image matrix shows the $\check{\boldsymbol{x}}$ map for the $T_{2}^{*}$-weighted image reconstruction in (24), and $\check{\boldsymbol{x}}_{R}$ and $\check{\boldsymbol{x}}_{I}$ maps for the joint $R_{2}^{*}$ and field map image reconstruction in (25). The $\check{\boldsymbol{x}}, \check{\boldsymbol{x}}_{R}$, and $\check{\boldsymbol{x}}_{I}$ maps were generated by respectively normalizing the estimated $\boldsymbol{x}, \boldsymbol{x}_{R}$ and $\boldsymbol{\kappa}_{I}$ maps with their spatial averages. The rows of the image matrix show the results across the penalized image reconstructions and the columns show them across the $\mathrm{k}$-space trajectories. 


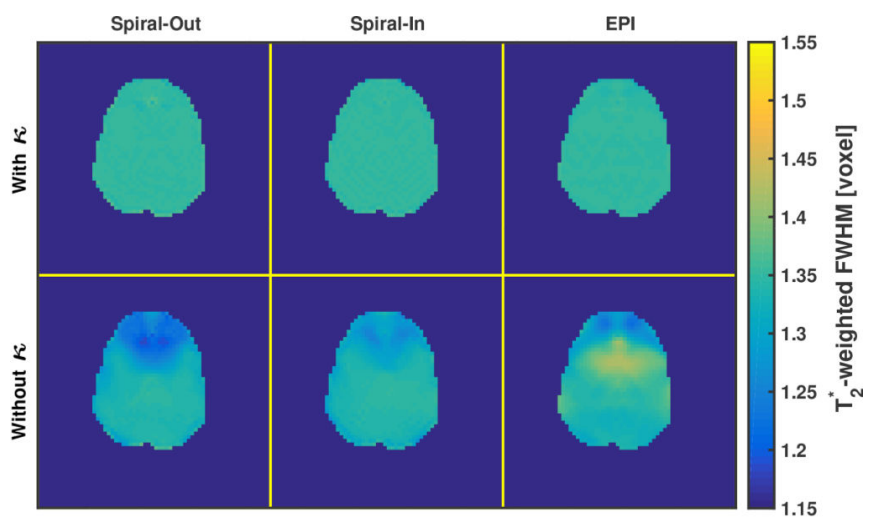

(a)

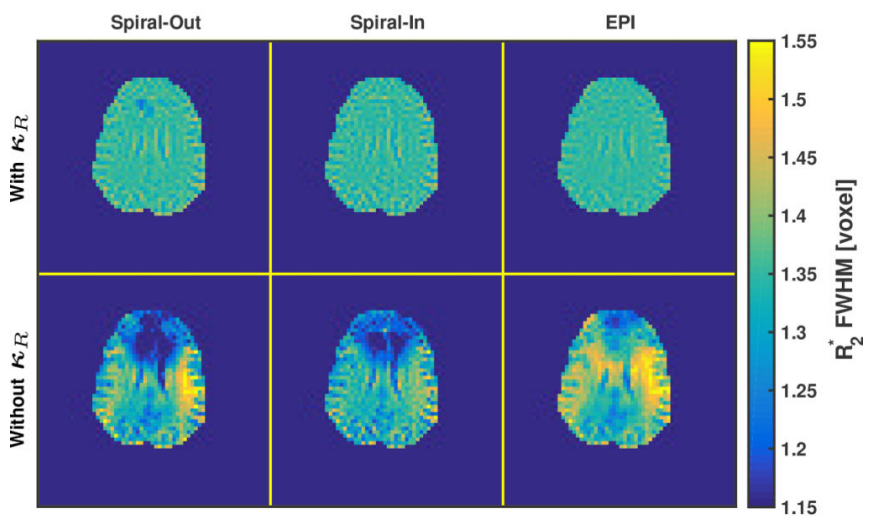

(b)

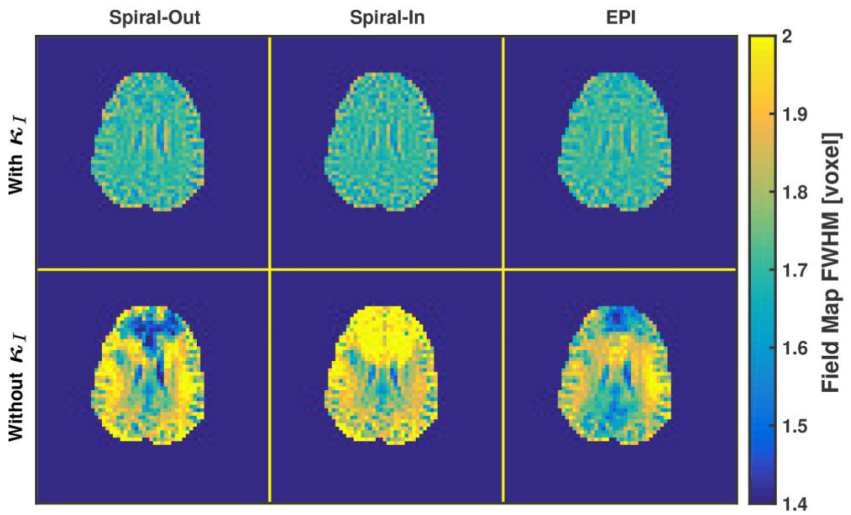

(c)

Fig. 7.

FWHM spatial maps for the (a) $T_{2}^{*}$-weighted, and joint (b) $R_{2}^{*}$ and (c) field map image reconstructions when using the spatially varying (top row) and non-varying (bottom row) roughness penalties. 